Supporting Information

\title{
When light and acid play tic-tac-toe with a nine-state
}

\section{molecular switch.}

\author{
Clément GUERRIN ${ }^{\dagger}$, Youssef AIDIBI ${ }^{\ddagger}$, Lionel SANGUINET ${ }^{\ddagger}$, Philippe LERICHE ${ }^{\ddagger}$, Stéphane ALOISE ${ }^{\dagger}$, Maylis \\ ORIO*§ ${ }^{\S}$ Stéphanie DELBAERE* ${ }^{\dagger}$ \\ ' Université de Lille, CNRS, UMR 8516, LASIR, Laboratoire de Spectrochimie Infra-rouge et Raman, F- \\ 59000 Lille, France \\ ${ }^{\ddagger}$ MOLTECH-Anjou, Université d'Angers, CNRS-UMR 6200, F-49045 Angers, France \\ ${ }^{\S}$ Aix Marseille Univ., CNRS, Centrale Marseille, iSm2, F-13397 Marseille, France
}

\section{Table of Content:}

Figure S1 : ${ }^{1} \mathrm{H}$ NMR spectra of BiBox-Phe in acetonitrile- $d_{3}$ upon addition of 1.0 and 3.0 eq. of acid. ....... 5 Figure S2: Switch from CC-tt and OO-tt with DCl addition and from OO-tt and CC-tt with $\mathrm{NEt}_{3}$ of BiBoxPhe in $\mathrm{CD}_{3} \mathrm{CN}$

Figure S3: Switch from CC-tt and OO-tt with $\mathrm{NOSbF}_{6}$ addition and from OO-tt and CC-tt with $\mathrm{NEt}_{3}$ of BiBox-Phe in $\mathrm{CD}_{3} \mathrm{CN}$...

Figure S4: Comparison of ${ }^{1} \mathrm{H}$ NMR spectra of OO-tt after addition of acid and oxidant of BiBox-Phe in $\mathrm{CD}_{3} \mathrm{CN}$

Figure S5: Switch from CC-tt and OO-tt with DCl addition and from OO-tt and CC-tt with $\mathrm{NEt}_{3}$ of BiBoxBithio in $\mathrm{CD}_{3} \mathrm{CN}$.

Figure S6: 1H NMR spectra of BiBox-Phe in OO-tt state (top) before and (bottom) after 6.5 minutes of $436 \mathrm{~nm}$ irradiation in $\mathrm{CDCl}_{3}$. (*) Solvent.

Figure S7 : ${ }^{1} \mathrm{H}$ NMR spectra of BiBox-Phe in OO-tt state (top) before and (bottom) after 5 minutes of 436

$\mathrm{nm}$ irradiation in acetonitrile- $\mathrm{d}_{3}$

Figure S8: ${ }^{1} \mathrm{H}$ NMR spectra of BiBox-Bithio in OO-tt form (top) before and (bottom) after 10 minutes of $546 \mathrm{~nm}$ irradiation in $\mathrm{CDCl}_{3}$. (*) Solvent.

Figure S9: ${ }^{1} \mathrm{H}$ NMR spectra of BiBox-Bithio in OO-tt form (top) before and (bottom) after 7 minutes of $577 \mathrm{~nm}$ irradiation in acetonitrile- $\mathrm{d}_{3}$

Figure S10 : ${ }^{1} \mathrm{H}$ NMR spectra of BiBox-Phe CC-tt upon $436 \mathrm{~nm}$ irradiation in acetonitrile- $\mathrm{d}_{3}$ 
Figure S11: Switch from CC-tt and CC-cc upon $577 \mathrm{~nm}$ irradiation and from CC-cc and CC-tt after thermal of BiBox-Bithio in $\mathrm{CD}_{3} \mathrm{CN}$

Figure S12: ${ }^{1} \mathrm{H}$ NMR spectra of (a) BiBox-Phe and (b) BiBox-Bithio in (top) CC-tt form, (middle) after addition of 1.5 equivalents of hydrochloric acid and (bottom) after visible light irradiation, 8 minutes at $436 \mathrm{~nm}$ and 5 minutes at $577 \mathrm{~nm}$ respectively in acetonitrile- $d_{3}$.

Figure S13: ${ }^{1} \mathrm{H}$ NMR spectra of (top) neat BiBox-Bithio in CC-tt form, (middle) after 232 minutes of 577 $\mathrm{nm}$ irradiation and (bottom) after addition of 1.5 equivalents of hydrochloric acid - in acetonitrile- $\mathrm{d}_{3} \ldots 17$ Figure S14: ${ }^{1} \mathrm{H}$ NMR spectra of (top) BiBox-Bithio in CC-tt form, (middle) after 10 minutes of $577 \mathrm{~nm}$ irradiation and (bottom) upon addition of a trifluoroacetic acid (TFA) vapor excess - in chloroform-d ... 18 Figure S15: Switch CC-tt $\rightarrow$ CC-cc (hv $577 \mathrm{~nm}$ ), CC-cc $\rightarrow$ OO-cc + OO-tc (TFA), OO-cc + OO-tc -tt $\rightarrow$ CC$\mathrm{cc}+\mathrm{CC}$-tc $\left(\mathrm{NEt}_{3}\right)$ of BiBox-Bithio in $\mathrm{CDIL}_{3}$

Figure S16: ${ }^{1} \mathrm{H}$ NMR spectrum of BiBox-Bithio CC-tt in acetonitrile- $\mathrm{d}_{3}$ at room temperature $-\left({ }^{*}\right)$ solvent -

$(\dagger)$ water..... 20

Figure S17: ${ }^{1} \mathrm{H}$ NMR spectrum of BiBox-Bithio CC-tc in acetonitrile- $\mathrm{d}_{3}$ at room temperature, obtained from CC-tt upon $577 \mathrm{~nm}$ irradiation (52 minutes) - In mixture with CC-tt $-\left({ }^{*}\right)$ solvent $-\left({ }^{\dagger}\right)$ water ...... 21 Figure S18: ${ }^{1} \mathrm{H}$ NMR spectrum of BiBox-Bithio CC-cc in acetonitrile- $\mathrm{d}_{3}$ at room temperature, obtained from CC-tt upon $577 \mathrm{~nm}$ irradiation (232 minutes) - In mixture with CC-tt and CC-tc $-\left({ }^{*}\right)$ solvent $-\left({ }^{\dagger}\right)$ water

Figure S19: ${ }^{1} \mathrm{H}$ NMR spectrum of BiBox-Bithio OC-tt in acetonitrile- $\mathrm{d}_{3}$ at room temperature, obtained from CC-tt upon addition of 1.5 eq. of $\mathrm{DCl}-$ In mixture with CC-tt and OO-tt $-\left({ }^{*}\right)$ solvent $-(\dagger)$ water ... 23 Figure S20: ${ }^{1} \mathrm{H}$ NMR spectrum of BiBox-Bithio OC-tc in acetonitrile- $\mathrm{d}_{3}$ at room temperature, obtained from OC-tt upon $577 \mathrm{~nm}$ irradiation (40 seconds) - In mixture with OC-tt and CC-tt - $\left(^{*}\right)$ solvent - $(+)$ water

Figure S21: ${ }^{1} \mathrm{H}$ NMR spectrum of BiBox-Bithio OC-cc in acetonitrile- $\mathrm{d}_{3}$ at room temperature, obtained from OC-tt upon $577 \mathrm{~nm}$ irradiation (5 minutes) - In mixture with OC-tt, OC-tc and CC-tt - $\left({ }^{*}\right)$ solvent $(+)$ water

Figure S22 : ${ }^{1} \mathrm{H}$ NMR spectrum of BiBox-Bithio OO-tt in acetonitrile- $\mathrm{d}_{3}$ at room temperature, obtained from CC-tt upon addition of TFA vapor excess $-\left({ }^{*}\right)$ solvent $-(+)$ water .

Figure S23 : ${ }^{1} \mathrm{H}$ NMR spectrum of BiBox-Bithio OO-tc in acetonitrile- $\mathrm{d}_{3}$ at room temperature, obtained from OO-tt upon $577 \mathrm{~nm}$ irradiation (1 minute) - in mixture with OO-tt and OO-cc - $\left({ }^{*}\right)$ solvent $-\left({ }^{\dagger}\right)$ water

Figure S24: ${ }^{1} \mathrm{H}$ NMR spectrum of BiBox-Bithio OO-cc in acetonitrile- $\mathrm{d}_{3}$ at room temperature, obtained from OO-tt upon $577 \mathrm{~nm}$ irradiation (7 minutes) - in mixture with OO-tt and OO-tc $-\left({ }^{*}\right)$ solvent $-\left({ }^{\dagger}\right)$ water

Figure S25: ${ }^{1} \mathrm{H}$ NMR spectrum of BiBox-Phe CC-tt in acetonitrile- $\mathrm{d}_{3}$ at room temperature $-\left({ }^{*}\right)$ solvent -

$(\dagger)$ water

Figure S26 : ${ }^{1} \mathrm{H}$ NMR spectrum of BiBox-Phe CC-tc in acetonitrile- $\mathrm{d}_{3}$ at room temperature, obtained from CC-tt upon $436 \mathrm{~nm}$ irradiation (70 minutes) - in mixture with CC-tt $-\left({ }^{*}\right)$ solvent $-\left({ }^{\dagger}\right)$ water . 30 Figure S27 : ${ }^{1} \mathrm{H}$ NMR spectrum of BiBox-Phe CC-cc in acetonitrile- $\mathrm{d}_{3}$ at room temperature, obtained from CC-tt upon $436 \mathrm{~nm}$ irradiation (265 minutes) - in mixture with CC-tt and CC-tc $-\left({ }^{*}\right)$ solvent $-\left({ }^{+}\right)$water 31 Figure S28: ${ }^{1} \mathrm{H}$ NMR spectrum of BiBox-Phe OC-tt in acetonitrile- $\mathrm{d}_{3}$ at room temperature, obtained from CC-tt upon addition of 1.5 eq. of $\mathrm{DCl}-$ in mixture with CC-tt and OO-tt $-(*)$ solvent $-(+)$ water 32 
Figure S29: ${ }^{1} \mathrm{H}$ NMR spectrum of BiBox-Phe OC-tc in acetonitrile- $\mathrm{d}_{3}$ at room temperature, obtained from OC-tt upon $436 \mathrm{~nm}$ irradiation (8 minutes) - in mixture with CC-tt, OC-tt and CC-tc $-\left({ }^{*}\right)$ solvent $-\left({ }^{\dagger}\right)$ water.

Figure S30: ${ }^{1} \mathrm{H}$ NMR spectrum of BiBox-Phe OO-tt in acetonitrile-d3 at room temperature, obtained from CC-tt upon addition of TFA vapor in excess $-(*)$ solvent $-(+)$ water .. 34

Figure S31: ${ }^{1} \mathrm{H}$ NMR spectrum of BiBox-Phe OO-tc in acetonitrile- $d_{3}$ at room temperature, obtained from OO-tt upon $436 \mathrm{~nm}$ irradiation (1.5 minutes) - in mixture with OO-tt and OO-cc $-\left({ }^{*}\right)$ solvent $-\left({ }^{\dagger}\right)$ water 35

Figure S32: ${ }^{1} \mathrm{H}$ NMR spectrum of BiBox-Phe OO-cc in acetonitrile- $\mathrm{d}_{3}$ at room temperature, obtained from OO-tt upon $436 \mathrm{~nm}$ irradiation (6.5 minutes) - in mixture with OO-tt and OO-tc $-\left({ }^{*}\right)$ solvent $-(\dagger)$ water 36

Figure S33 : Variation of the thermal rate constants of relaxation of BiBox $: c c \rightarrow t c=f(t c \rightarrow t t)$ 37 


\section{Characteristic NMR data used as probes to identify}

- the closed and open states of Box unit

- the trans and cis configuration of ethylenic bond

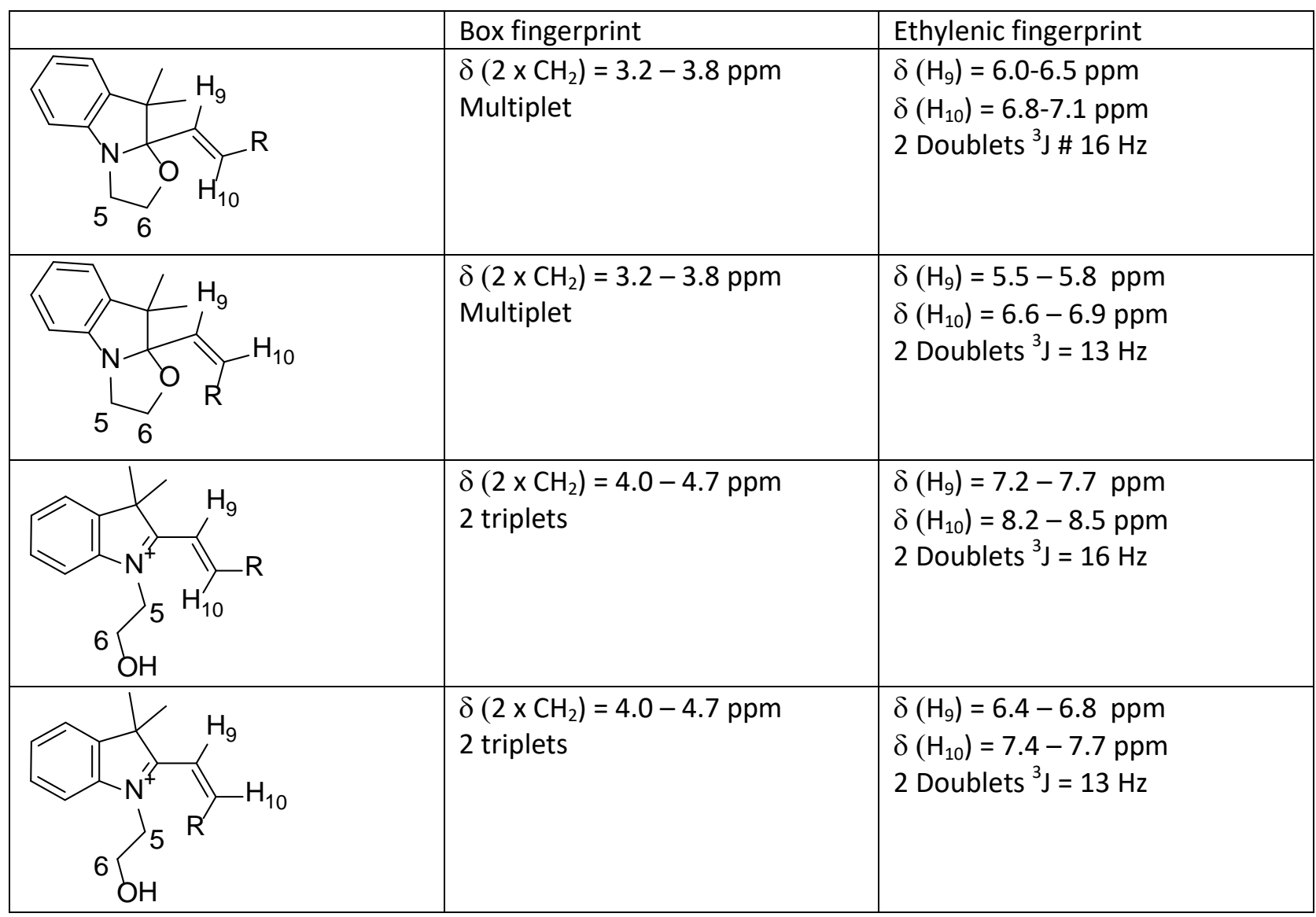



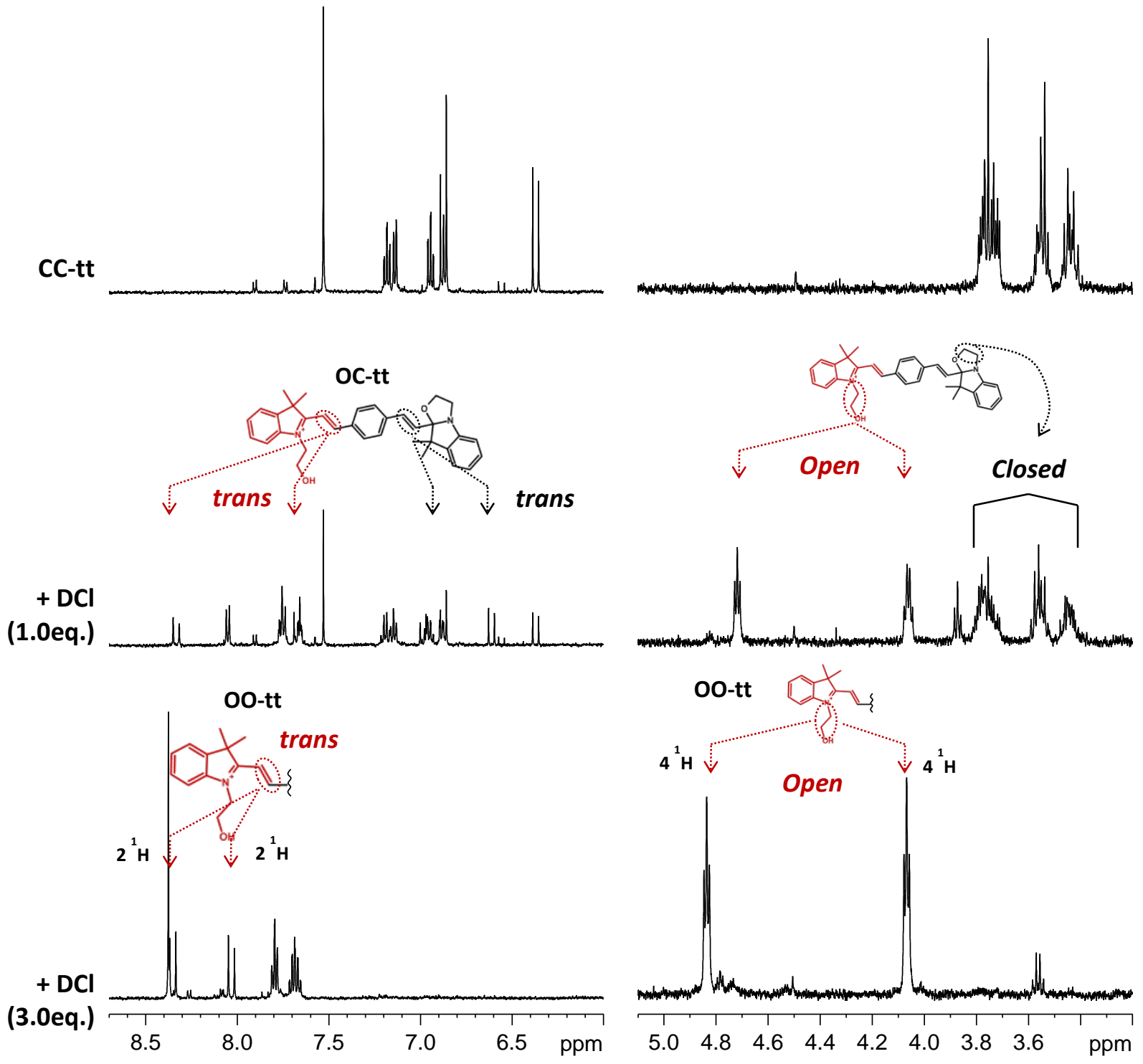

Figure S1 : ${ }^{1} \mathrm{H}$ NMR spectra of BiBox-Phe in acetonitrile- $\mathrm{d}_{3}$ upon addition of 1.0 and 3.0 eq. of acid. 


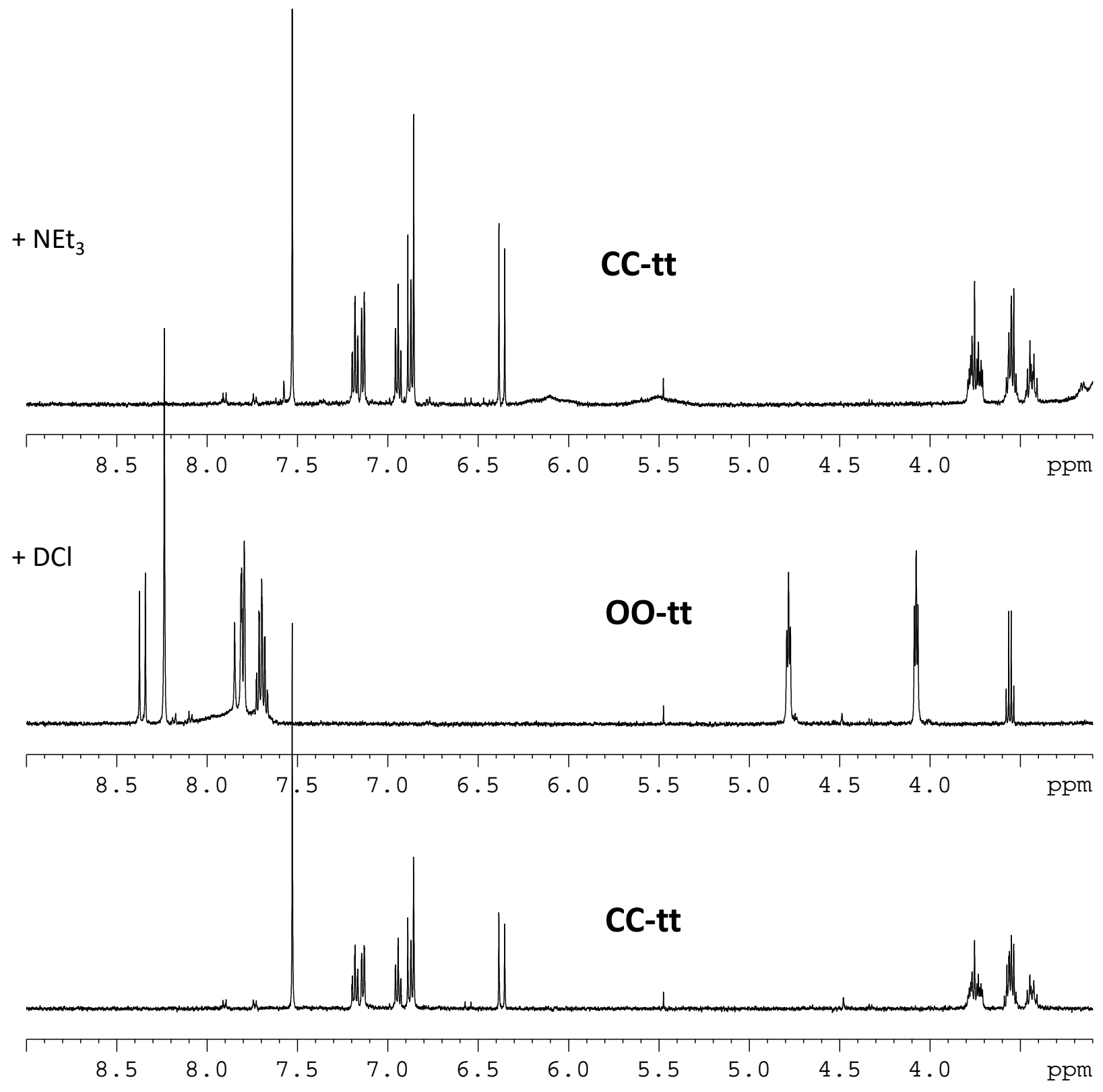

Figure S2: Switch from CC-tt and OO-tt with DCl addition and from OO-tt and CC-tt with $\mathrm{NEt}_{3}$ of BiBox-Phe in $\mathrm{CD}_{3} \mathrm{CN}$ 


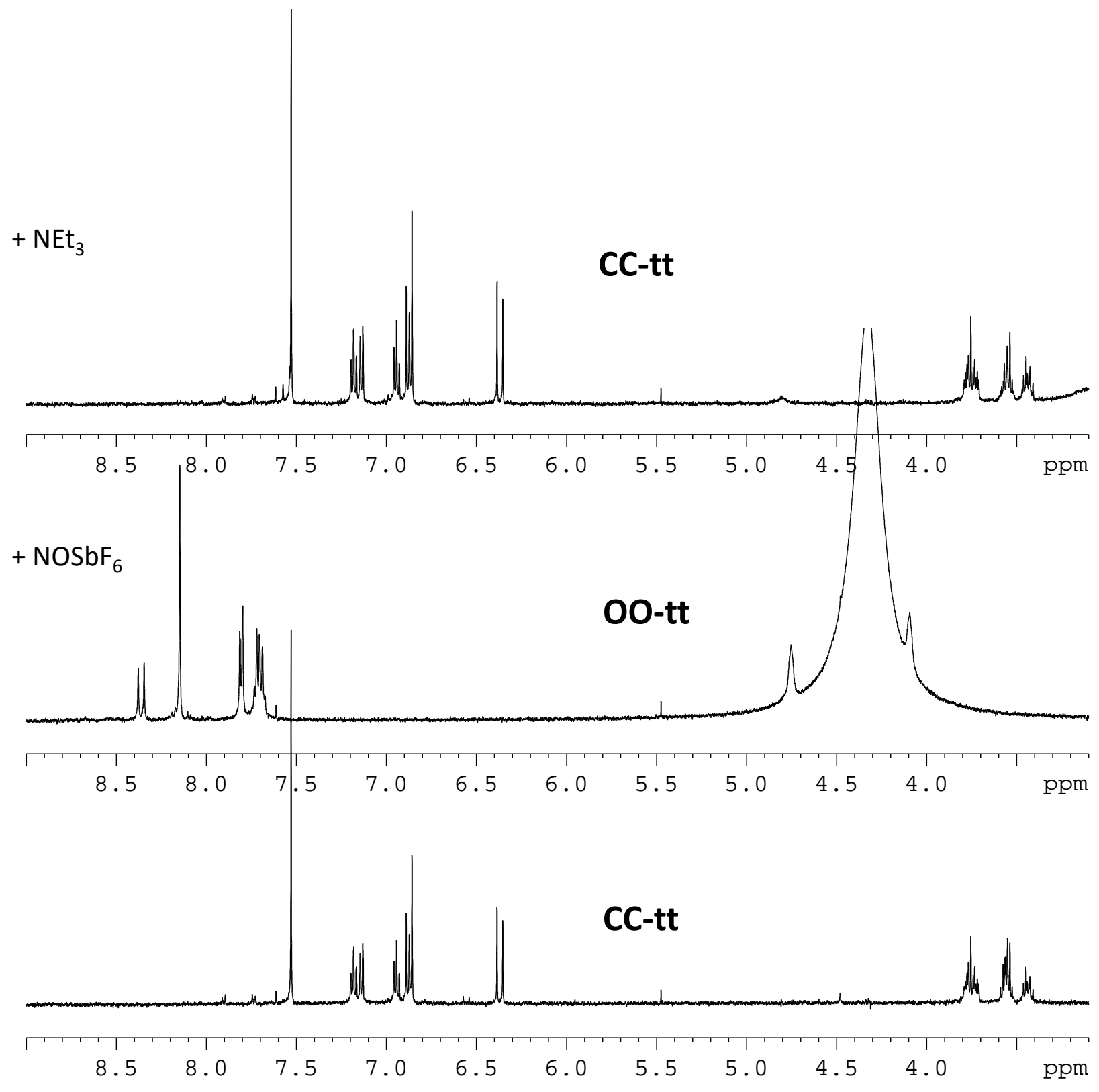

Figure S3: Switch from CC-tt and OO-tt with NOSbF 6 addition and from OO-tt and CC-tt with NEt ${ }_{3}$ of BiBox-Phe in $\mathrm{CD}_{3} \mathrm{CN}$ 


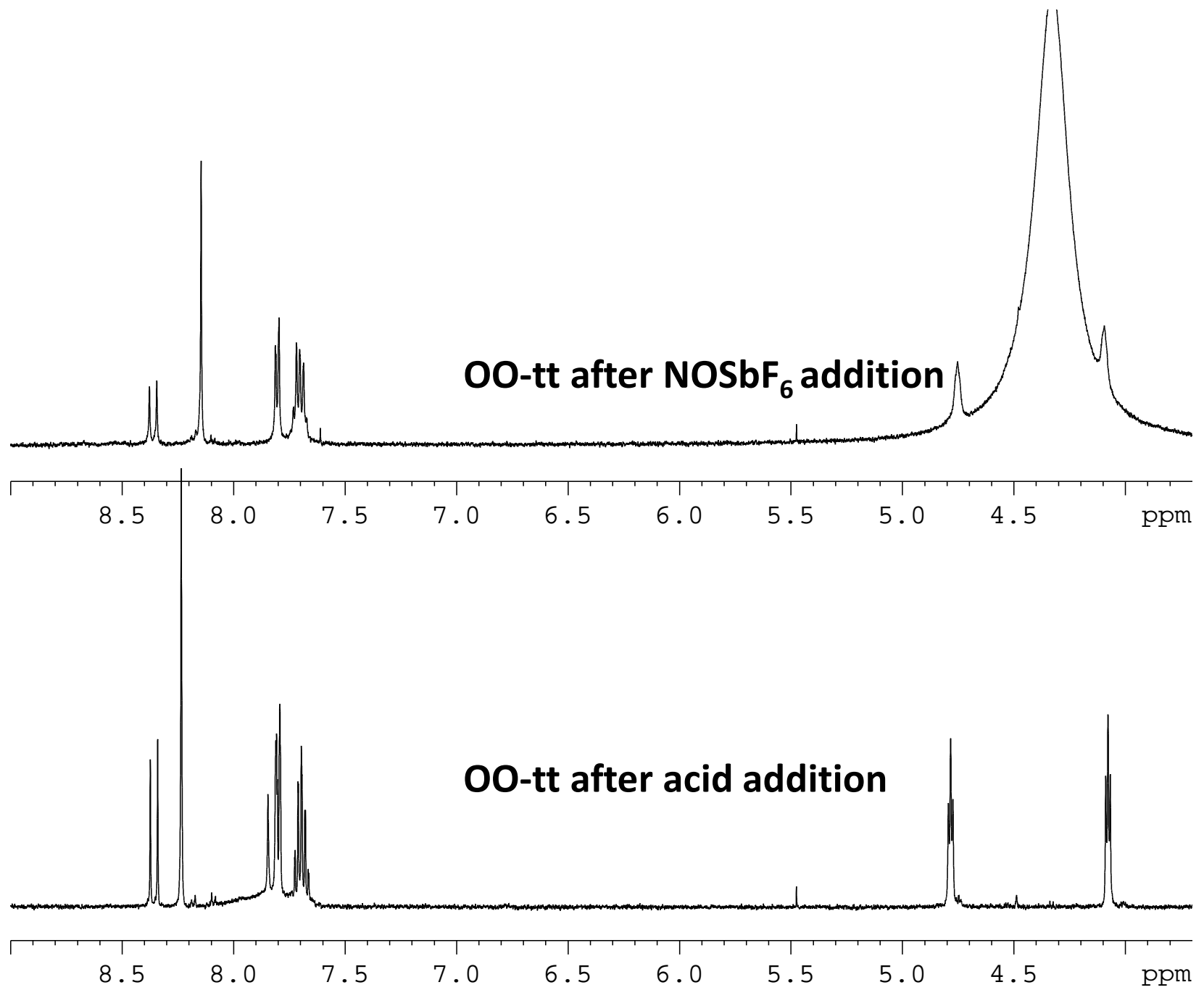

Figure S4: Comparison of ${ }^{1} \mathrm{H}$ NMR spectra of OO-tt after addition of acid and oxidant of BiBox-Phe in $\mathrm{CD}_{3} \mathrm{CN}$ 


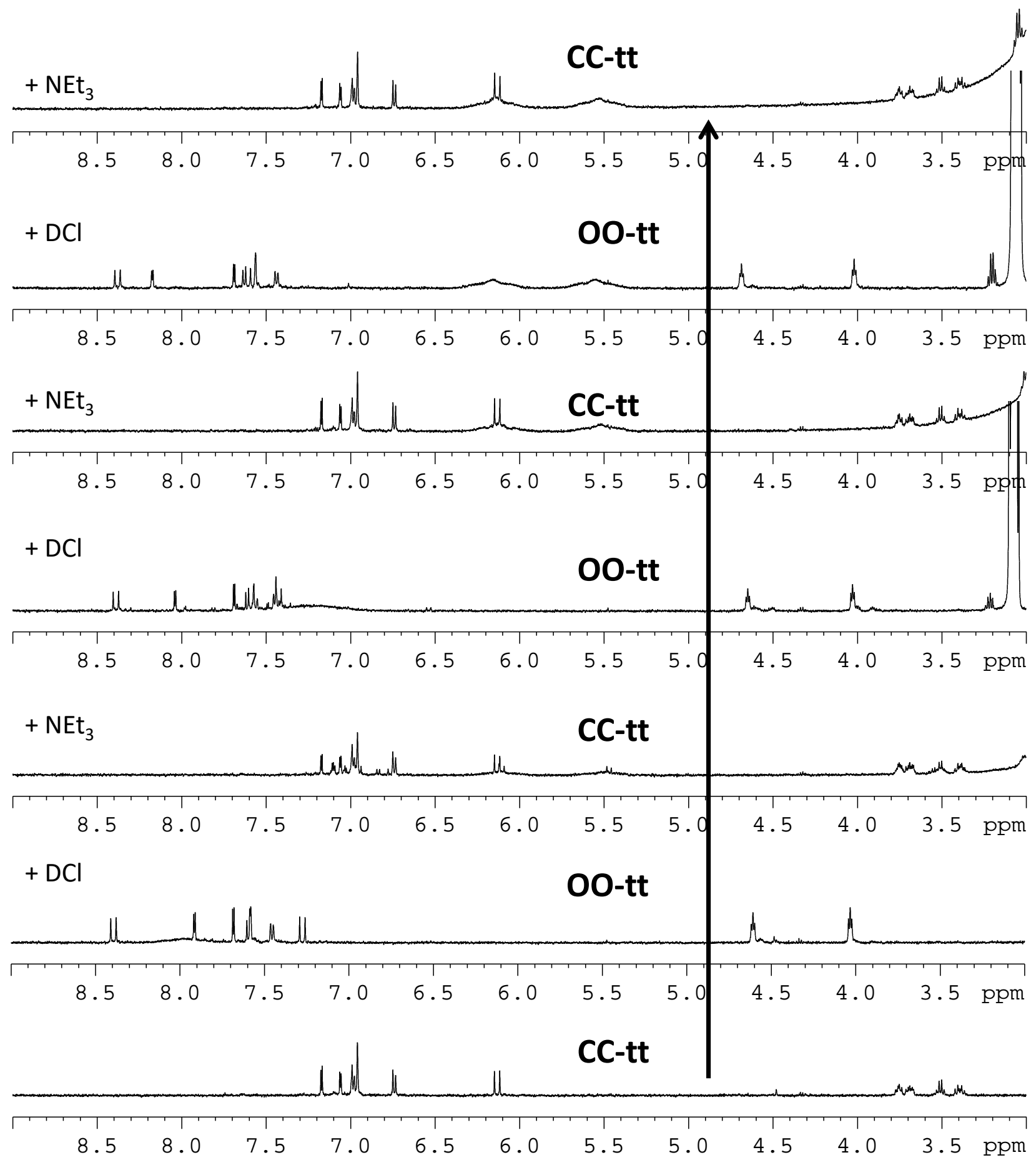

Figure S5: Switch from CC-tt and OO-tt with DCl addition and from OO-tt and CC-tt with NEt ${ }_{3}$ of BiBox-Bithio in $\mathrm{CD}_{3} \mathrm{CN}$ 


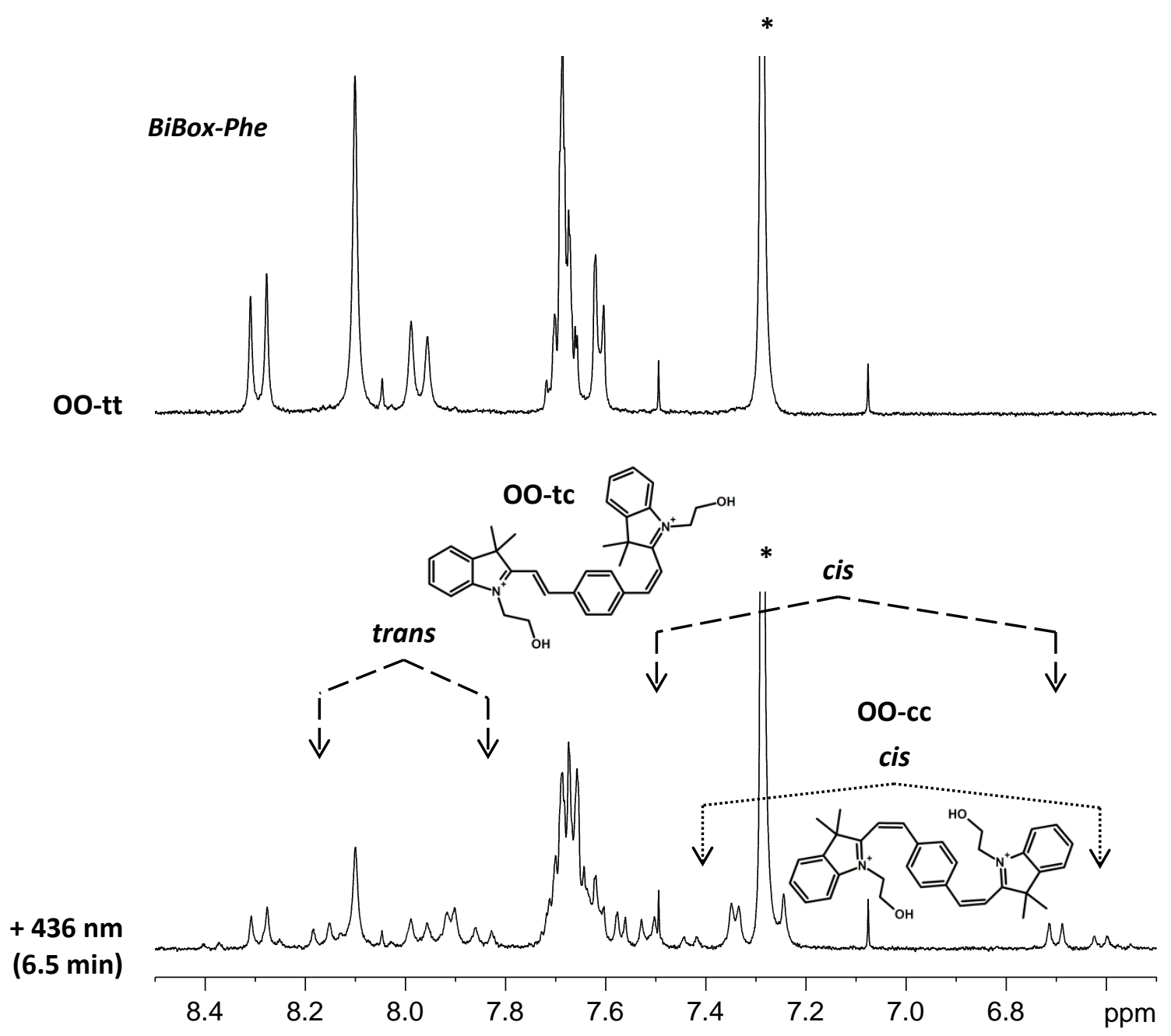

Figure S6: $1 \mathrm{H}$ NMR spectra of BiBox-Phe in 0O-tt state (top) before and (bottom) after 6.5 minutes of $436 \mathrm{~nm}$ irradiation in $\mathrm{CDCl}_{3}$. (*) Solvent. 


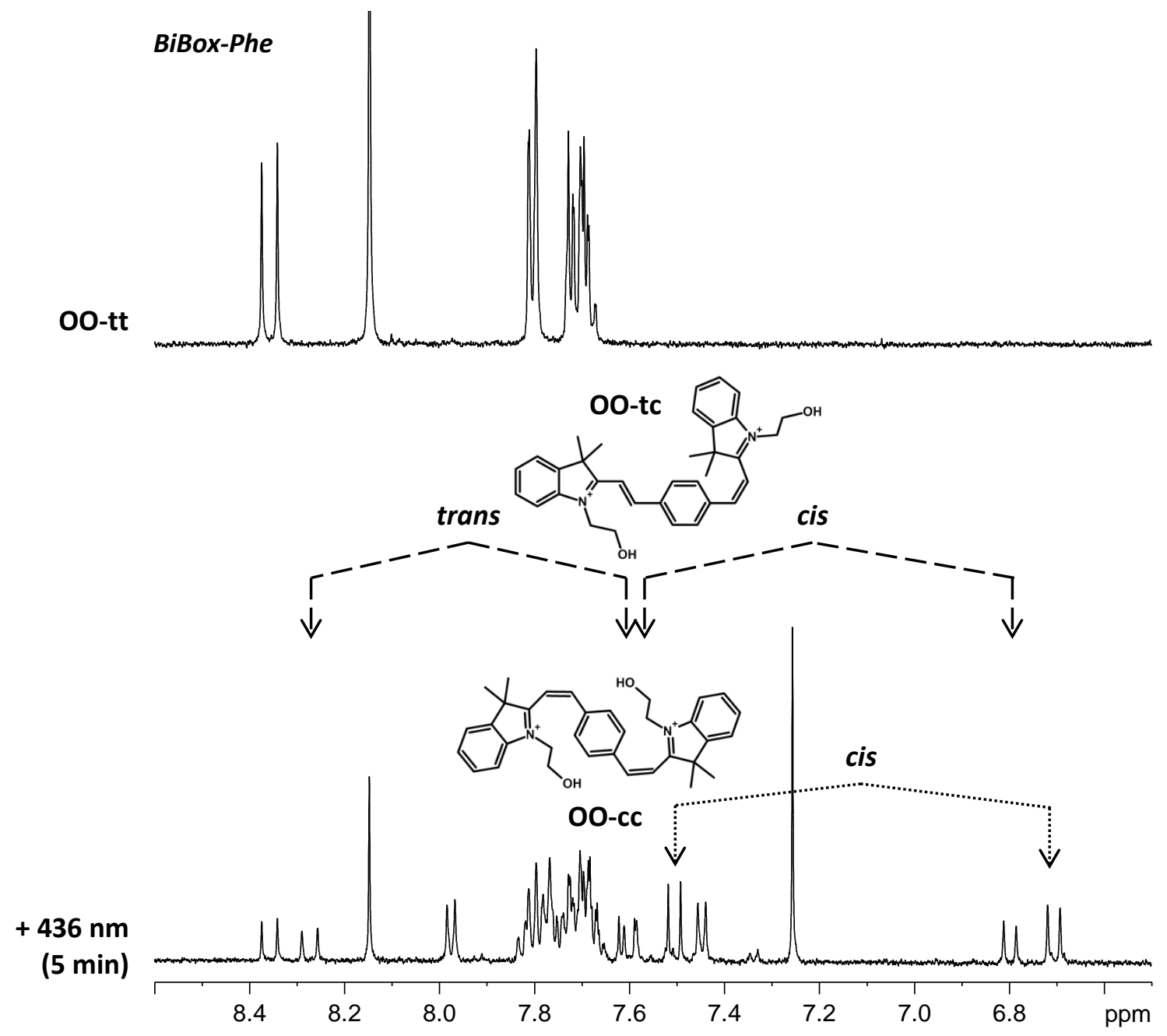

Figure S7 : ${ }^{1} \mathrm{H}$ NMR spectra of BiBox-Phe in OO-tt state (top) before and (bottom) after 5 minutes of $436 \mathrm{~nm}$ irradiation in acetonitrile- $d_{3}$ 

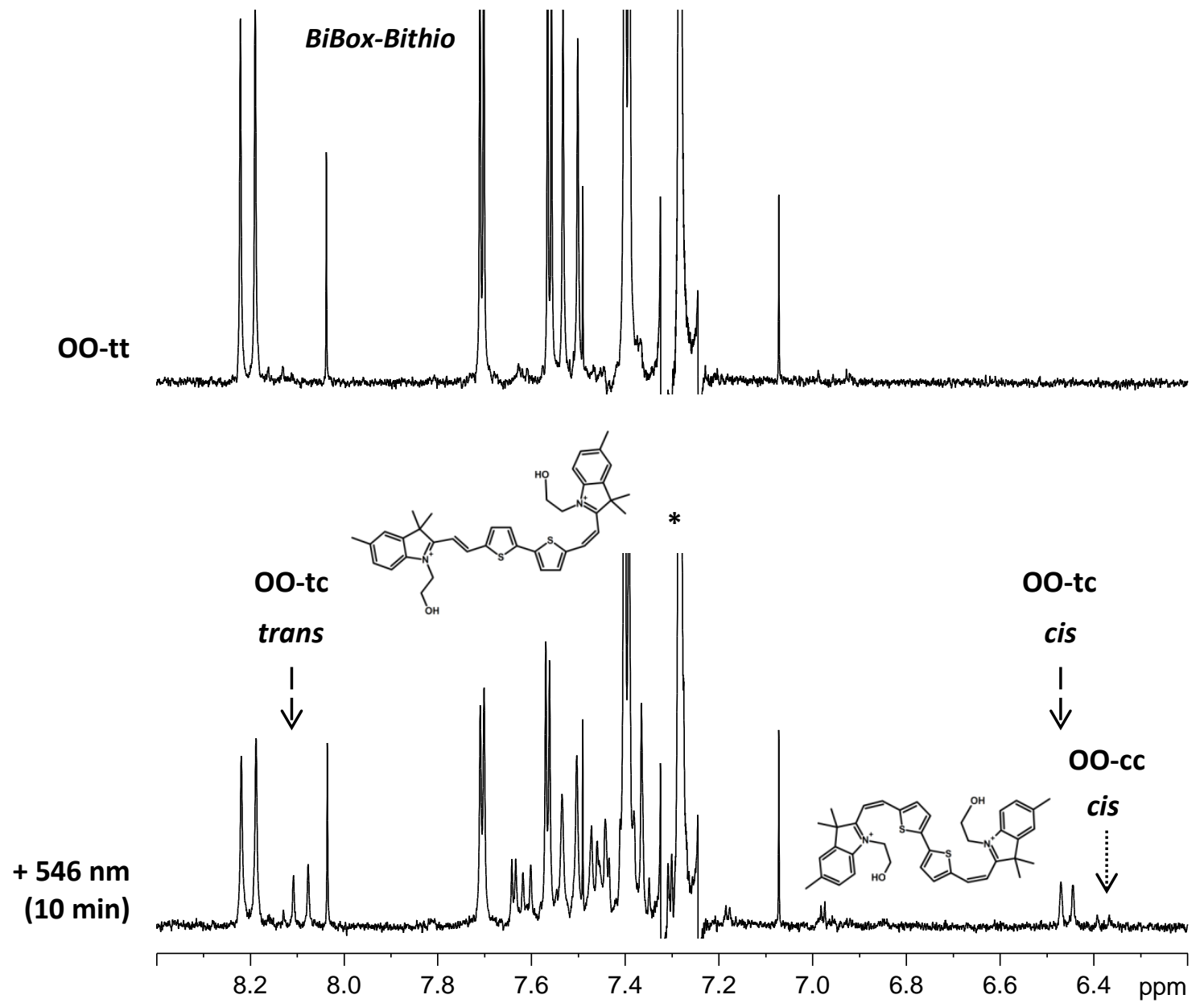

Figure S8: ${ }^{1} \mathrm{H}$ NMR spectra of BiBox-Bithio in OO-tt form (top) before and (bottom) after 10 minutes of $546 \mathrm{~nm}$ irradiation in $\mathrm{CDCl}_{3} .\left(^{*}\right)$ Solvent. 


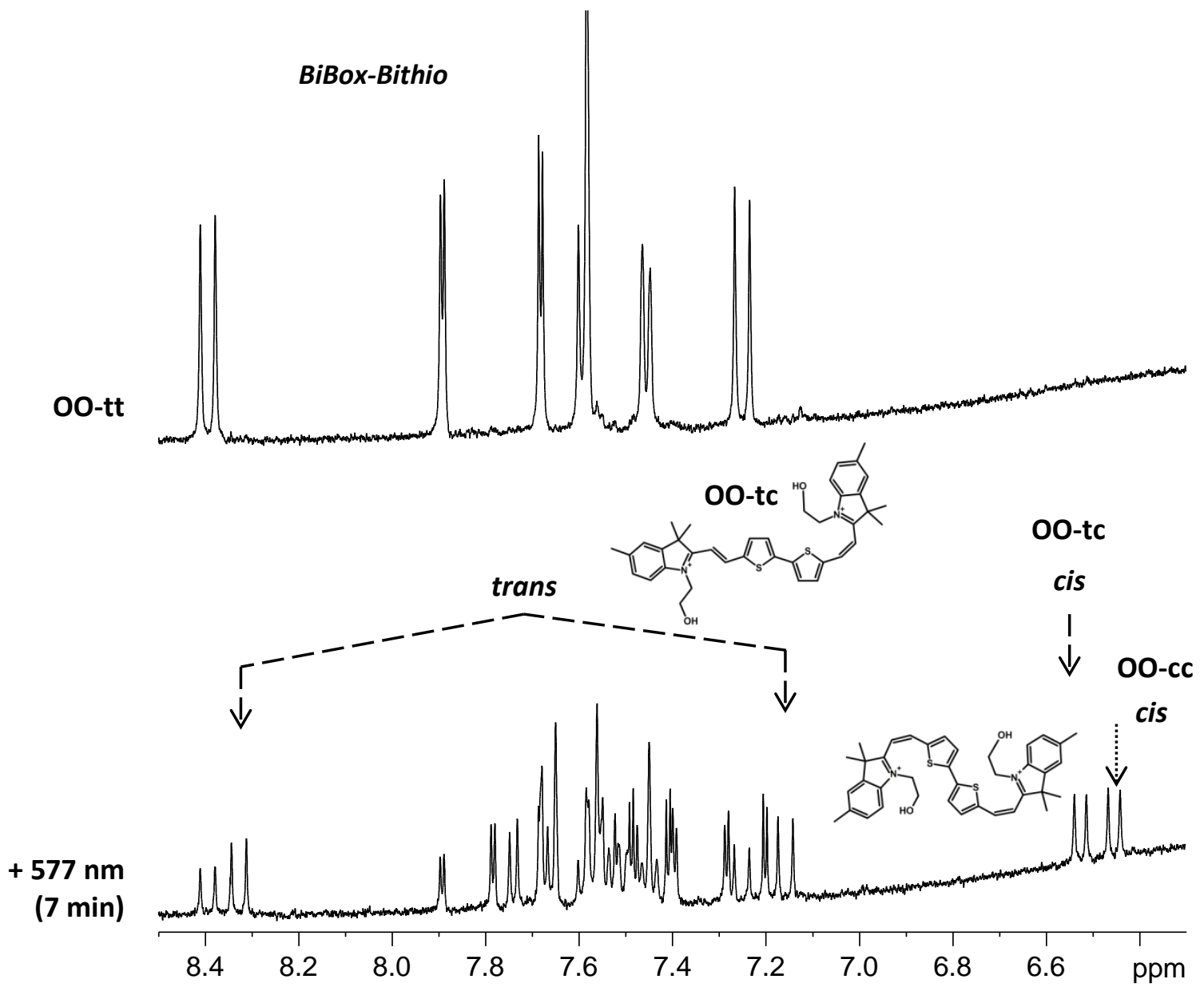

Figure S9: ${ }^{1} \mathrm{H}$ NMR spectra of BiBox-Bithio in 00-tt form (top) before and (bottom) after 7 minutes of $577 \mathrm{~nm}$ irradiation in acetonitrile- $d_{3}$. 

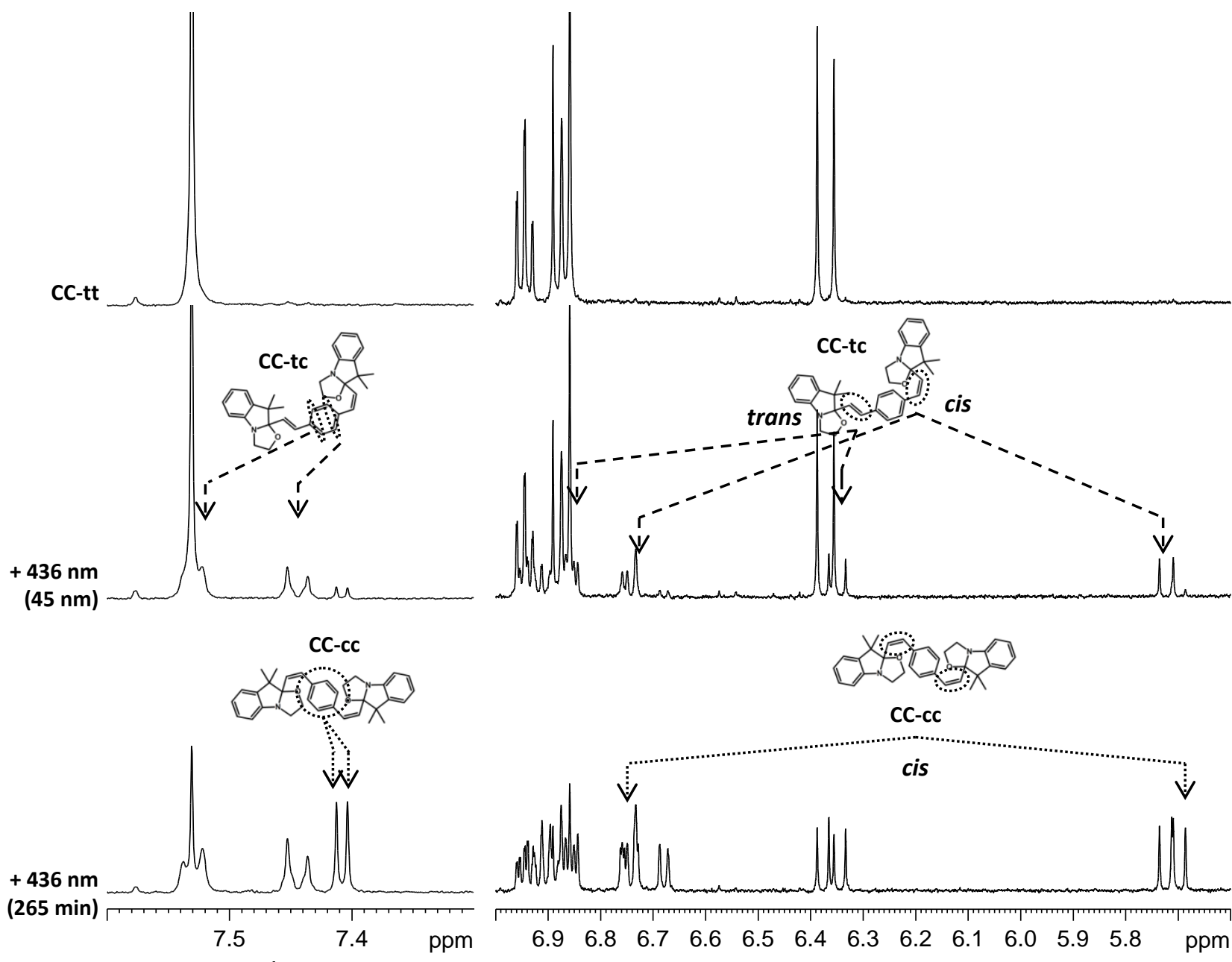

Figure S10 : ${ }^{1} \mathrm{H}$ NMR spectra of BiBox-Phe CC-tt upon $436 \mathrm{~nm}$ irradiation in acetonitrile-d $\mathrm{d}_{3}$. 


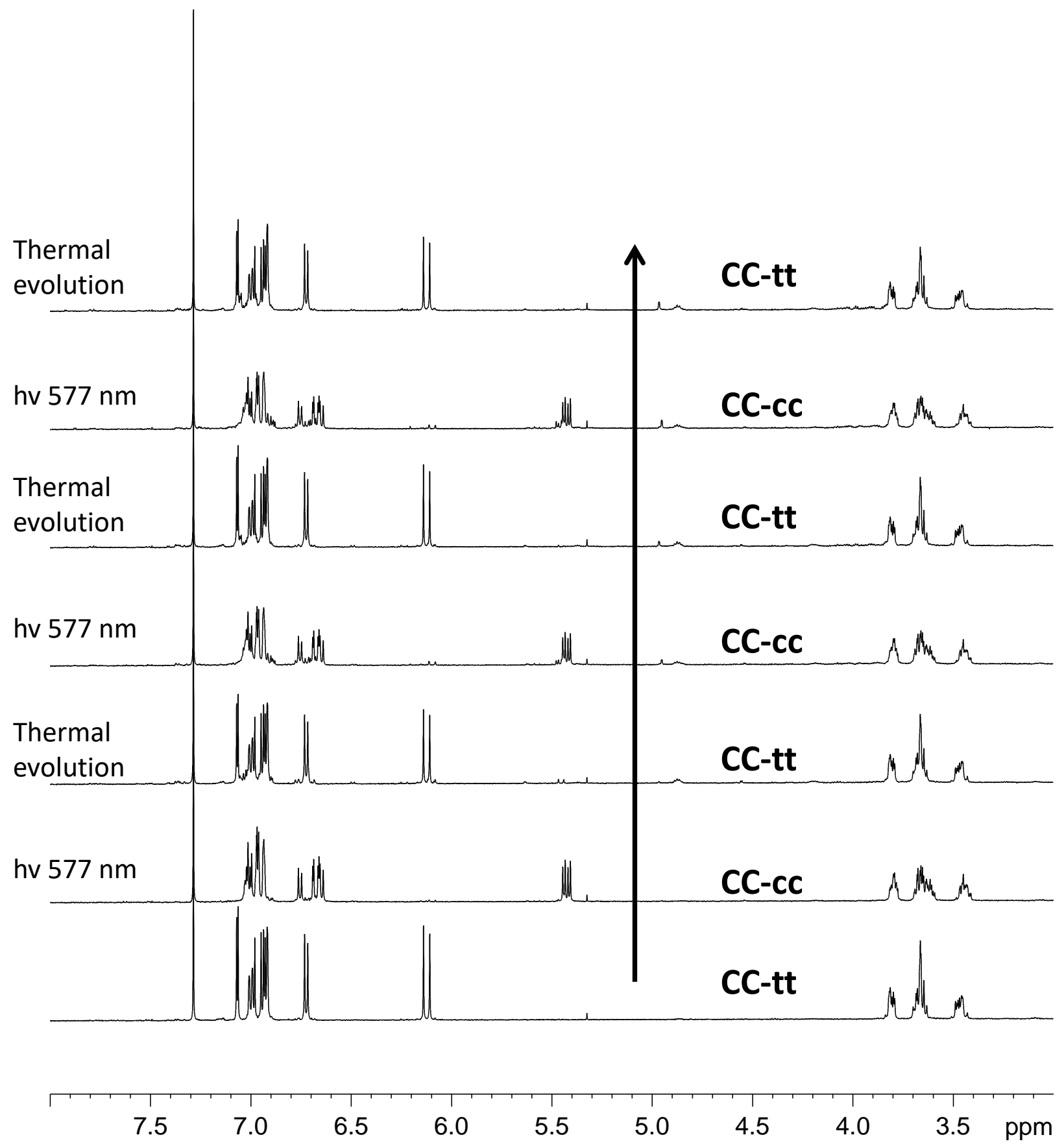

Figure S11: Switch from CC-tt and CC-cc upon $577 \mathrm{~nm}$ irradiation and from CC-cc and CC-tt after thermal of BiBox-Bithio in $\mathrm{CD}_{3} \mathrm{CN}$ 

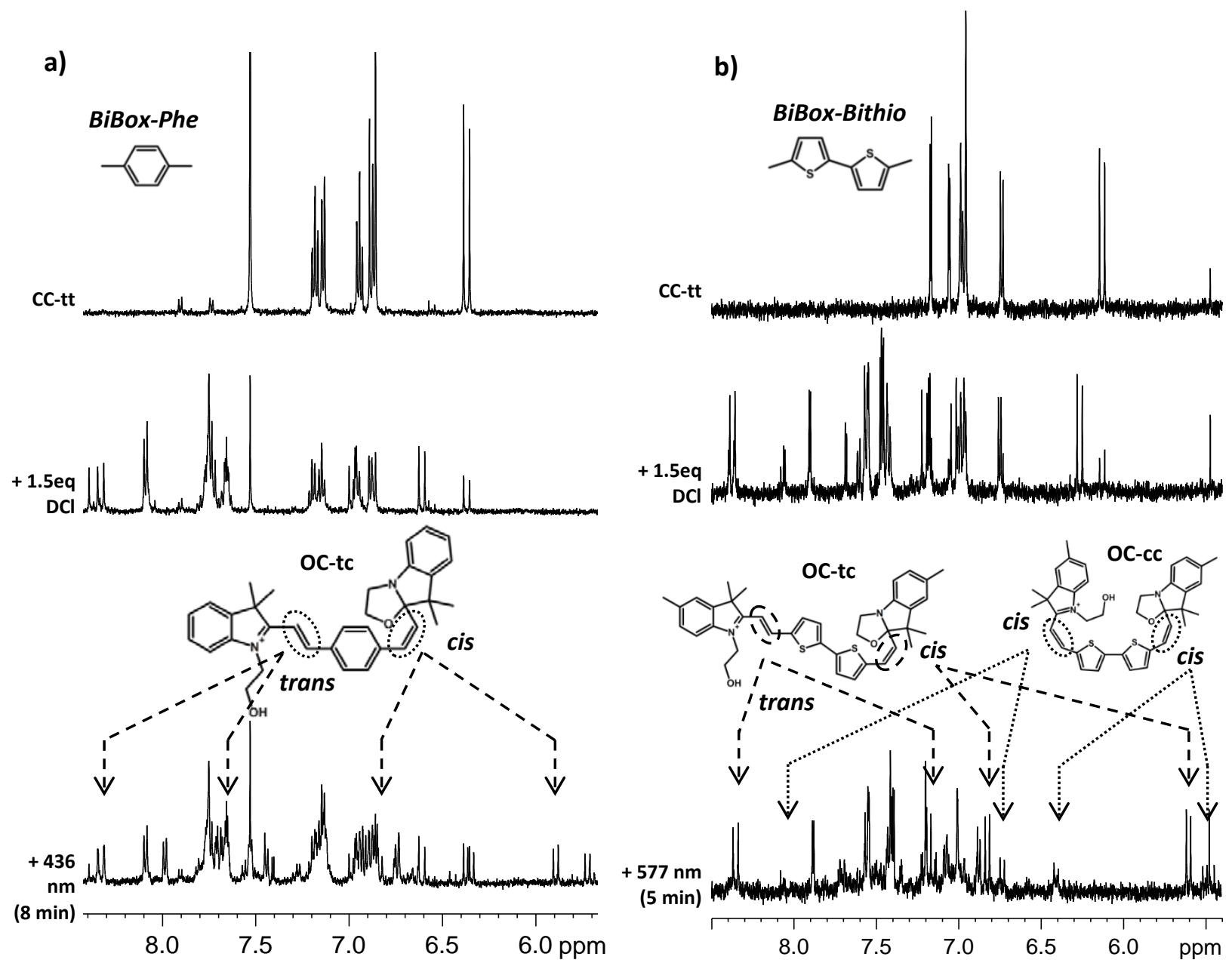

Figure S12: ${ }^{1} \mathrm{H}$ NMR spectra of (a) BiBox-Phe and (b) BiBox-Bithio in (top) CC-tt form, (middle) after addition of 1.5 equivalents of hydrochloric acid and (bottom) after visible light irradiation, 8 minutes at $436 \mathrm{~nm}$ and $5 \mathrm{minutes}$ at $577 \mathrm{~nm}$ respectively in acetonitrile- $d_{3}$. 


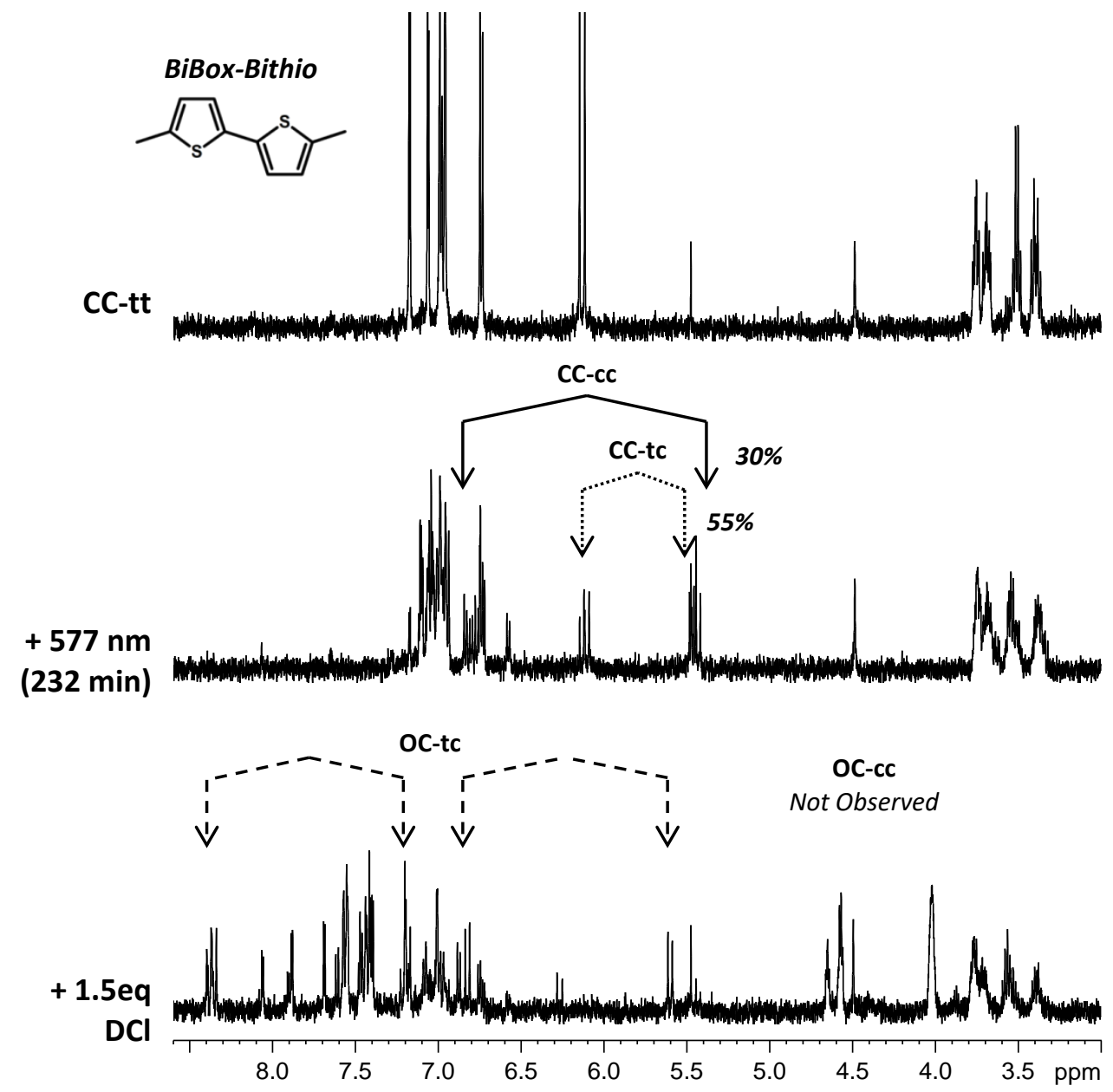

Figure S13 : ${ }^{1} \mathrm{H}$ NMR spectra of (top) neat BiBox-Bithio in CC-tt form, (middle) after 232 minutes of $577 \mathrm{~nm}$ irradiation and (bottom) after addition of 1.5 equivalents of hydrochloric acid - in acetonitrile- $d_{3}$ 


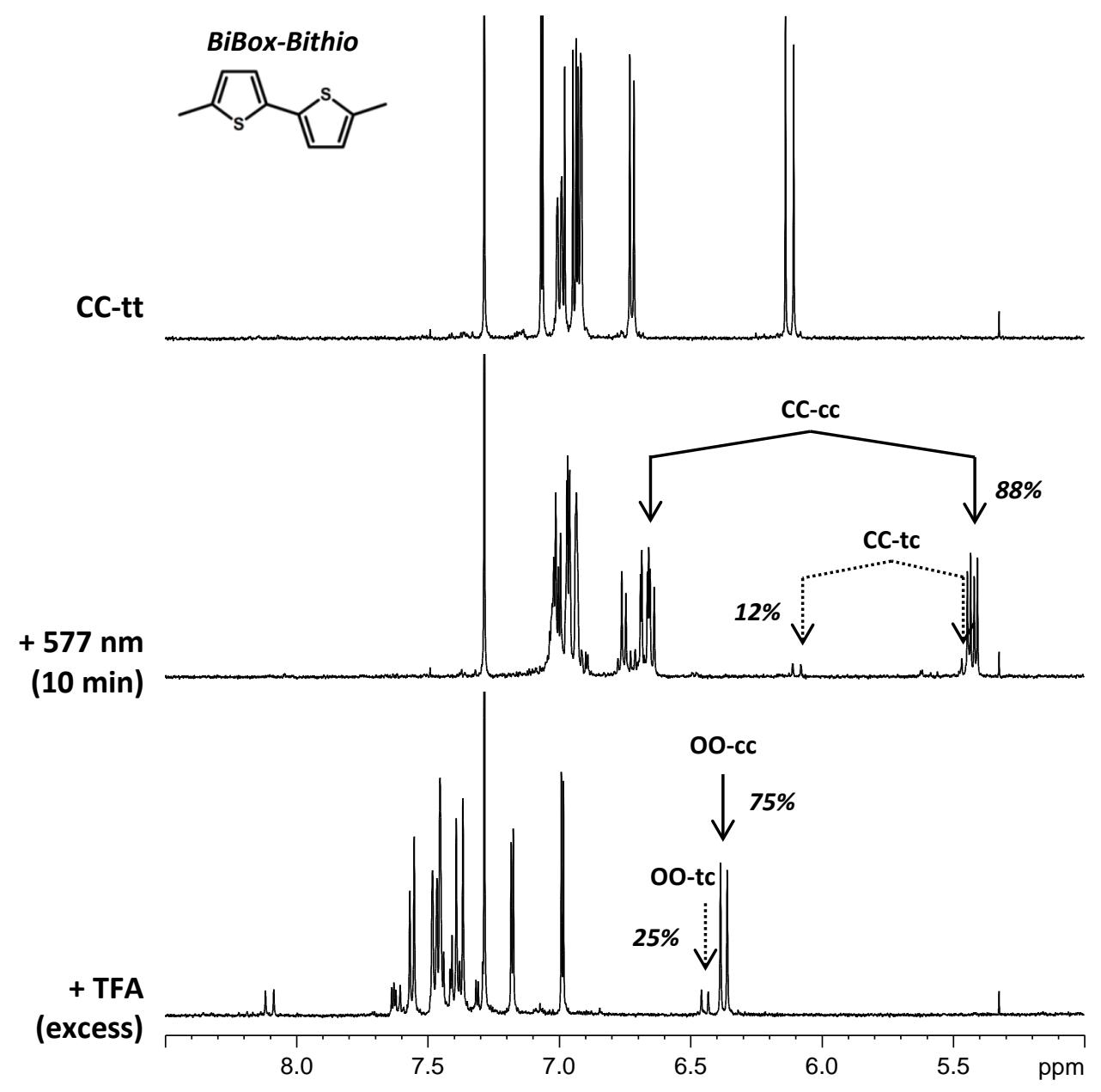

Figure S14: ${ }^{1} \mathrm{H}$ NMR spectra of (top) BiBox-Bithio in CC-tt form, (middle) after 10 minutes of $577 \mathrm{~nm}$ irradiation and (bottom) upon addition of a trifluoroacetic acid (TFA) vapor excess - in chloroform-d 


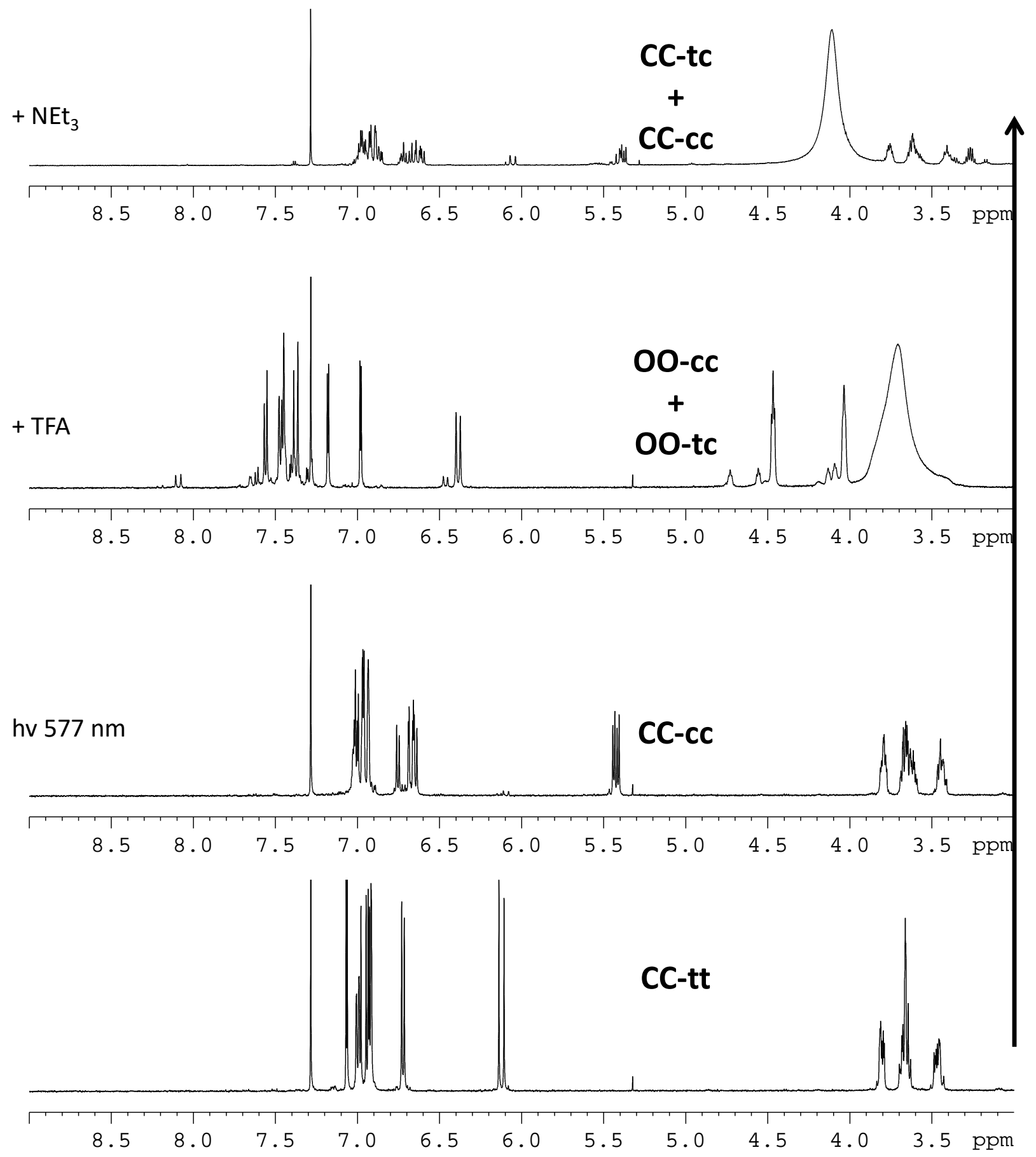

Figure S15: CC-tt $\rightarrow$ CC-cc (hv 577 nm), CC-cc $\rightarrow$ OO-cc + OO-tc (TFA), OO-cc + OO-tc -tt $\rightarrow$ CC-cc+CC-tc $\left(\mathrm{NEt}_{3}\right)$ of BiBox-Bithio in $\mathrm{CDIL}_{3}$ 


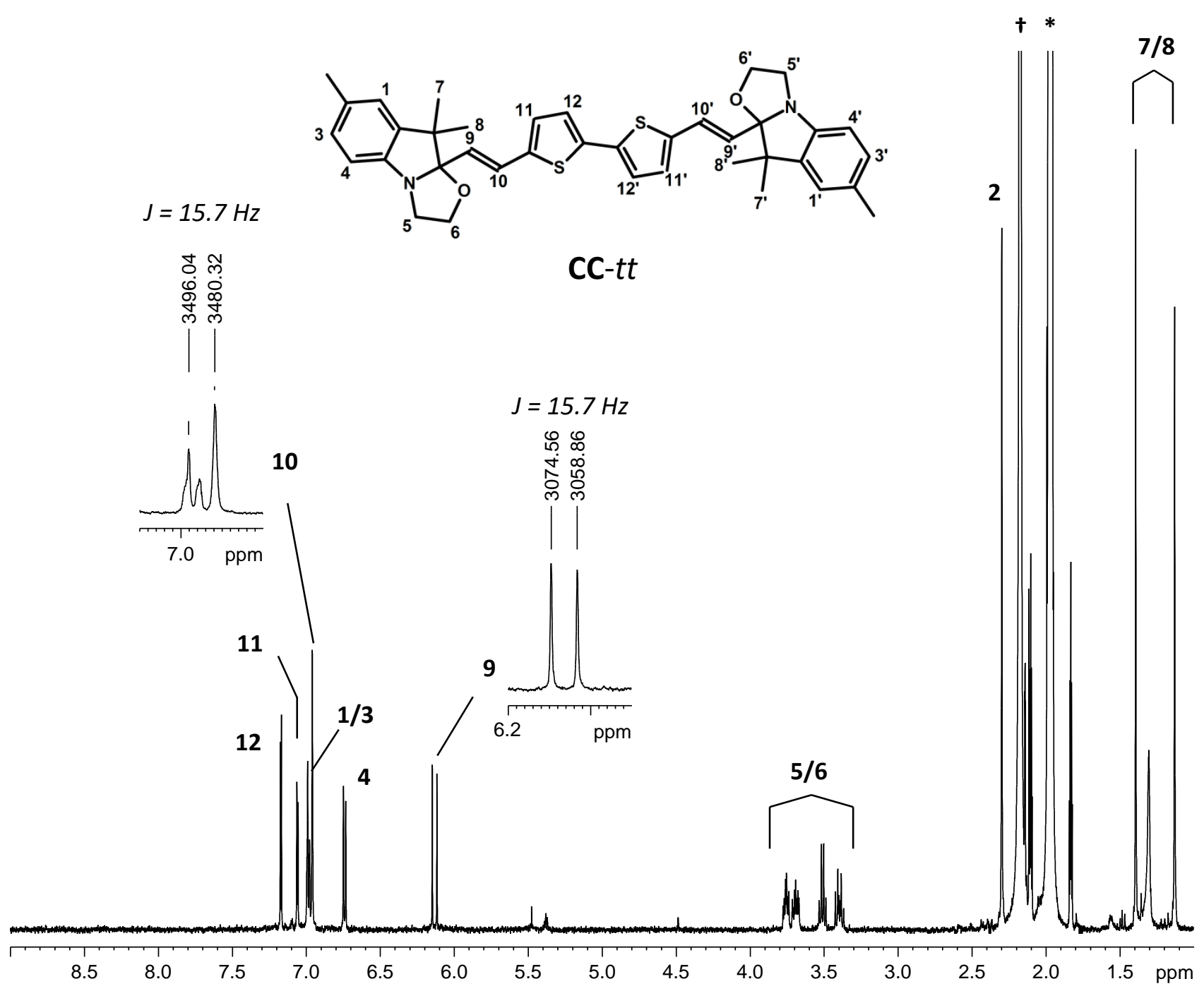

Figure S16: ${ }^{1} \mathrm{H}$ NMR spectrum of BiBox-Bithio CC-tt in acetonitrile- $d_{3}$ at room temperature $-(*)$ solvent $-(t)$ water 


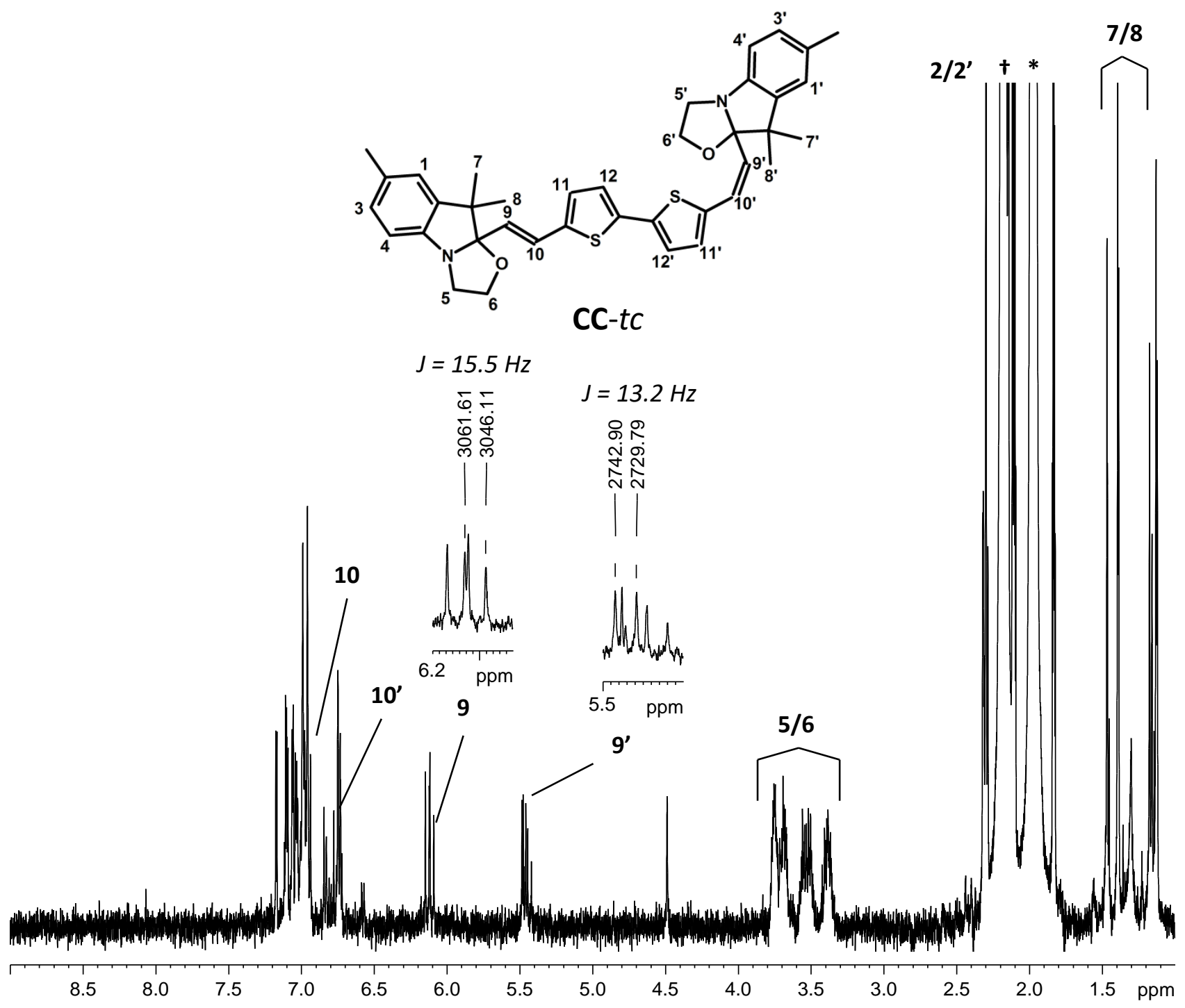

Figure S17: ${ }^{1} \mathrm{H}$ NMR spectrum of BiBox-Bithio CC-tc in acetonitrile- $\mathrm{d}_{3}$ at room temperature, obtained from CC-tt upon $577 \mathrm{~nm}$ irradiation (52 minutes) - In mixture with CC-tt $-(*)$ solvent $-(t)$ water 

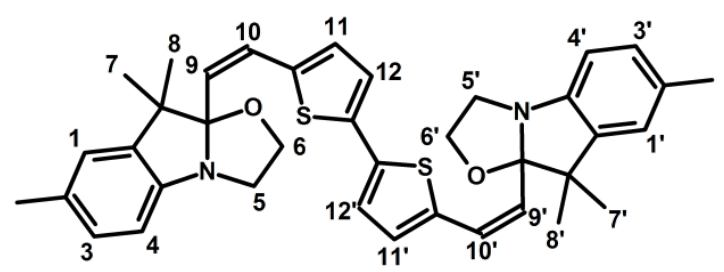

CC-cC

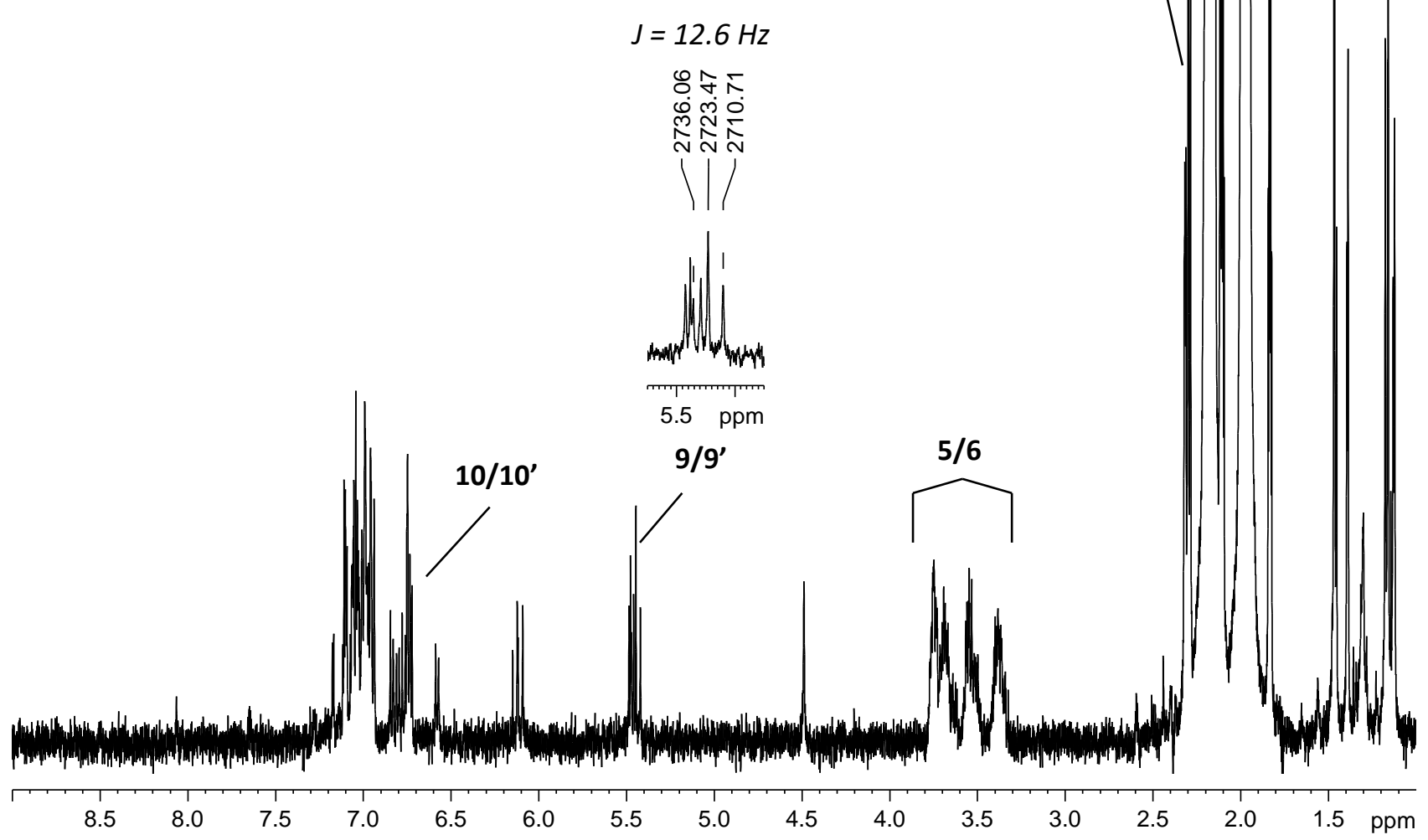

Figure S18 : ${ }^{1} \mathrm{H}$ NMR spectrum of BiBox-Bithio CC-cc in acetonitrile- $\mathrm{d}_{3}$ at room temperature, obtained from CC-tt upon $577 \mathrm{~nm}$ irradiation (232 minutes) - In mixture with CC-tt and CC-tc $-\left({ }^{*}\right)$ solvent $-(t)$ water 


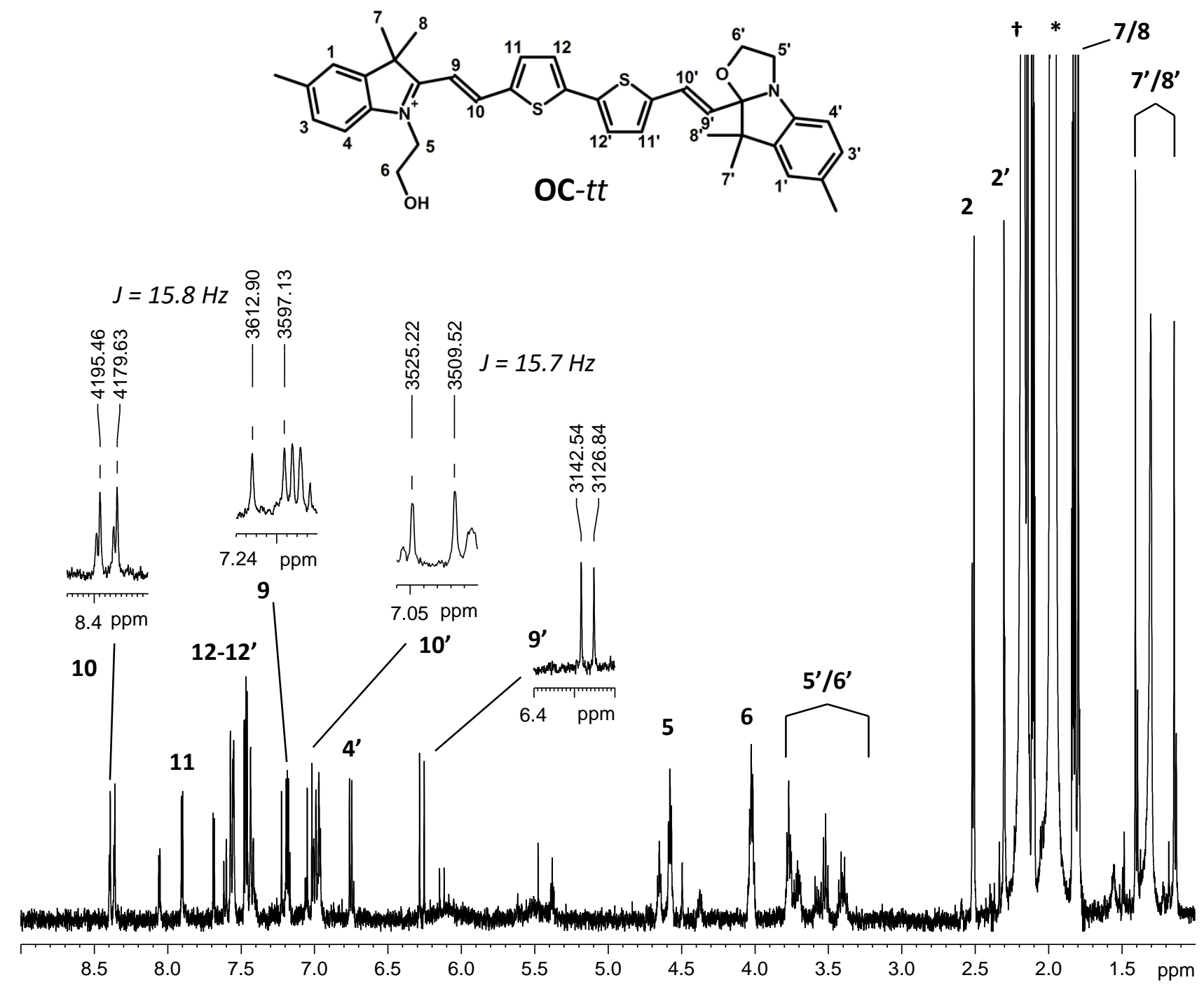

Figure S19 : ${ }^{1} \mathrm{H}$ NMR spectrum of BiBox-Bithio OC-tt in acetonitrile- $\mathrm{d}_{3}$ at room temperature, obtained from CC-tt upon addition of 1.5 eq. of $\mathrm{DCl}-$ In mixture with CC-tt and OO-tt $-(*)$ solvent $-(t)$ water 


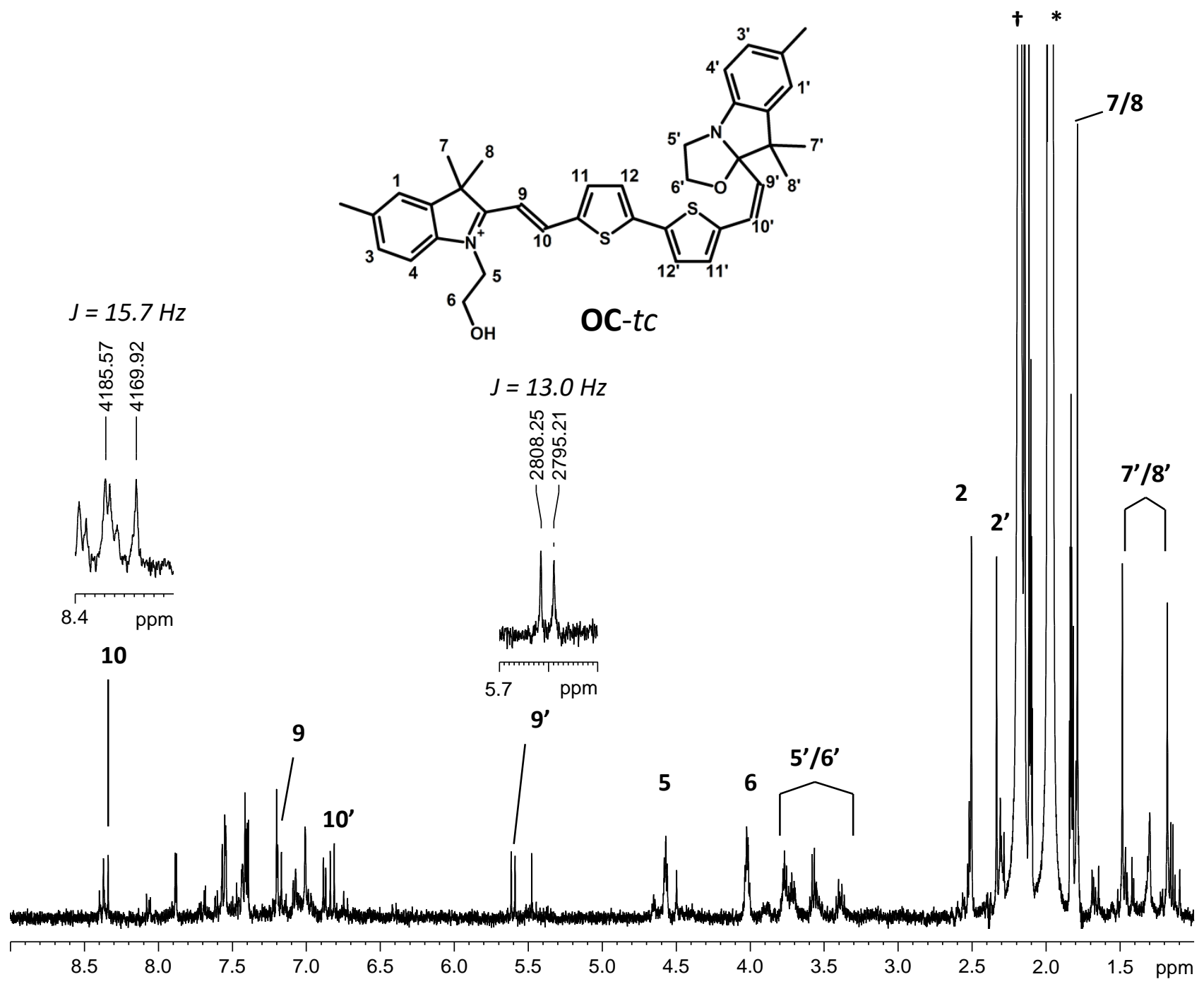

Figure S20 : ${ }^{1} \mathrm{H}$ NMR spectrum of BiBox-Bithio OC-tc in acetonitrile- $\mathrm{d}_{3}$ at room temperature, obtained from OC-tt upon $577 \mathrm{~nm}$ irradiation (40 seconds) - In mixture with OC-tt and CC-tt $-(*)$ solvent $-(t)$ water 

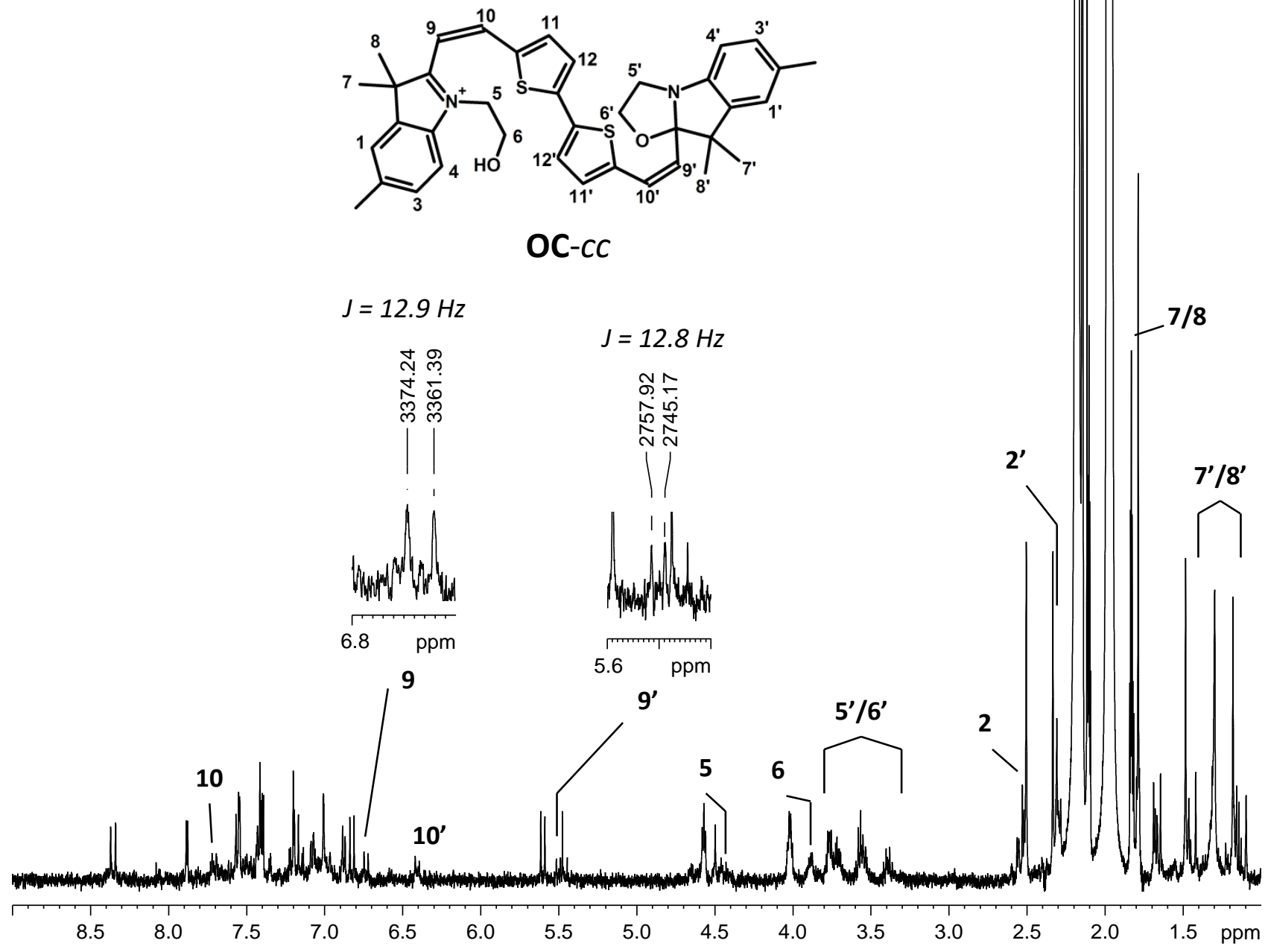

Figure S21 : ${ }^{1} \mathrm{H}$ NMR spectrum of BiBox-Bithio OC-cc in acetonitrile-d $\mathrm{d}_{3}$ at room temperature, obtained from OC-tt upon 577 $\mathrm{nm}$ irradiation (5 minutes) - In mixture with OC-tt, OC-tc and CC-tt $-(*)$ solvent $-(t)$ water 

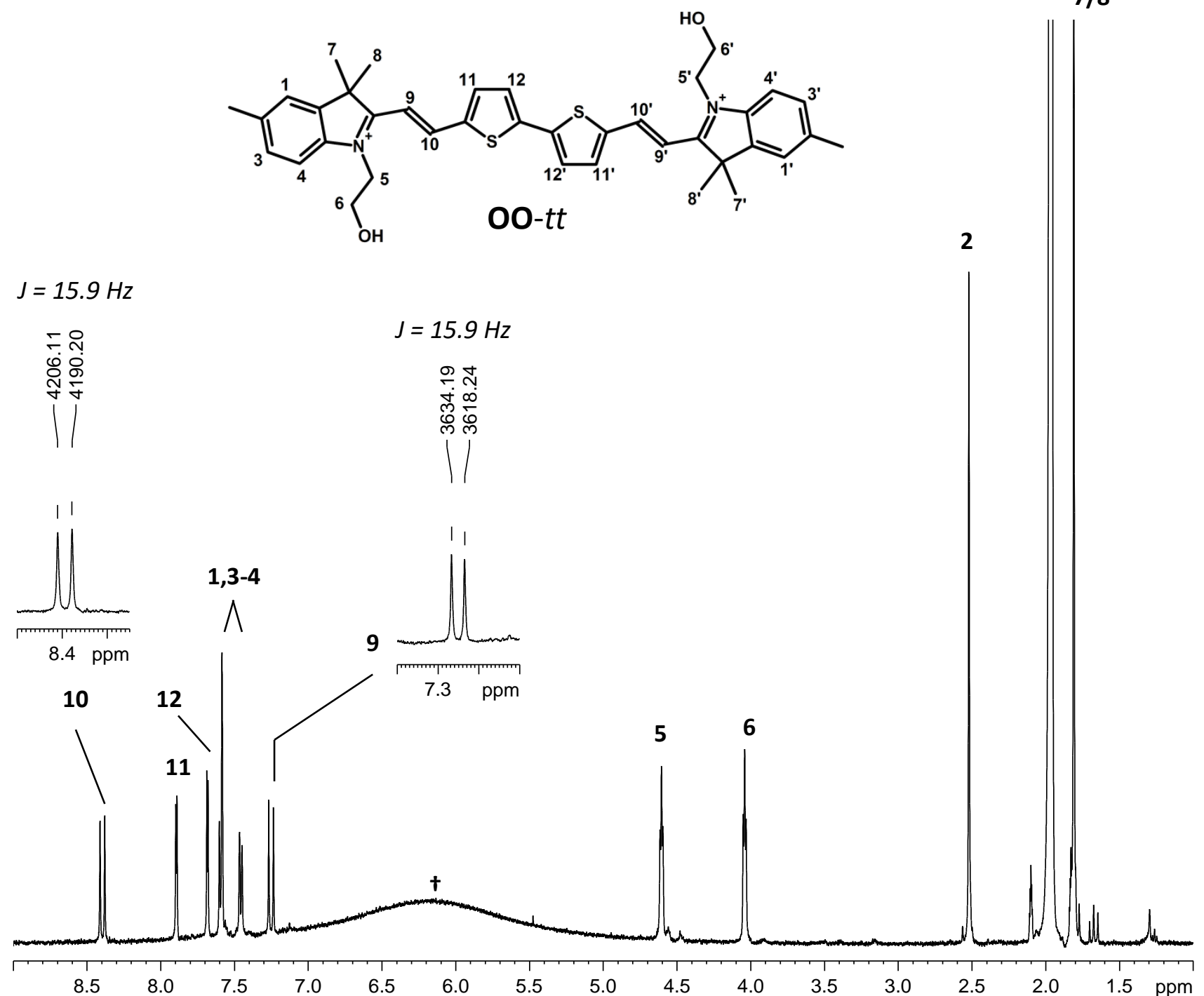

Figure S22 : ${ }^{1} \mathrm{H}$ NMR spectrum of BiBox-Bithio OO-tt in acetonitrile- $\mathrm{d}_{3}$ at room temperature, obtained from CC-tt upon addition of TFA vapor excess $-(*)$ solvent $-(+)$ water 


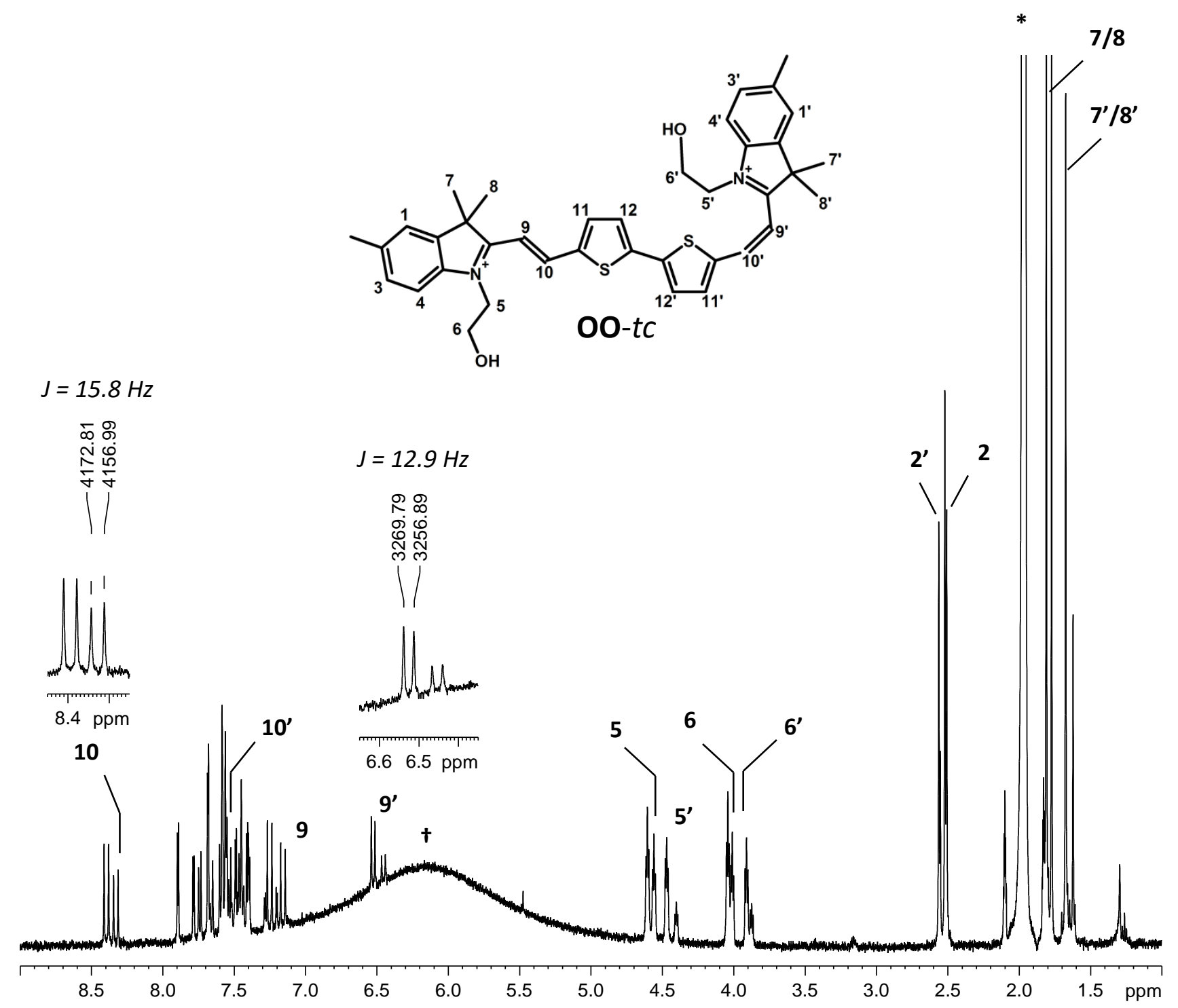

Figure S23 : ${ }^{1} \mathrm{H}$ NMR spectrum of BiBox-Bithio 00-tc in acetonitrile-d $\mathrm{d}_{3}$ at room temperature, obtained from 00-tt upon 577 $\mathrm{nm}$ irradiation (1 minute) - in mixture with OO-tt and OO-cc $-(*)$ solvent $-(\dagger)$ water 

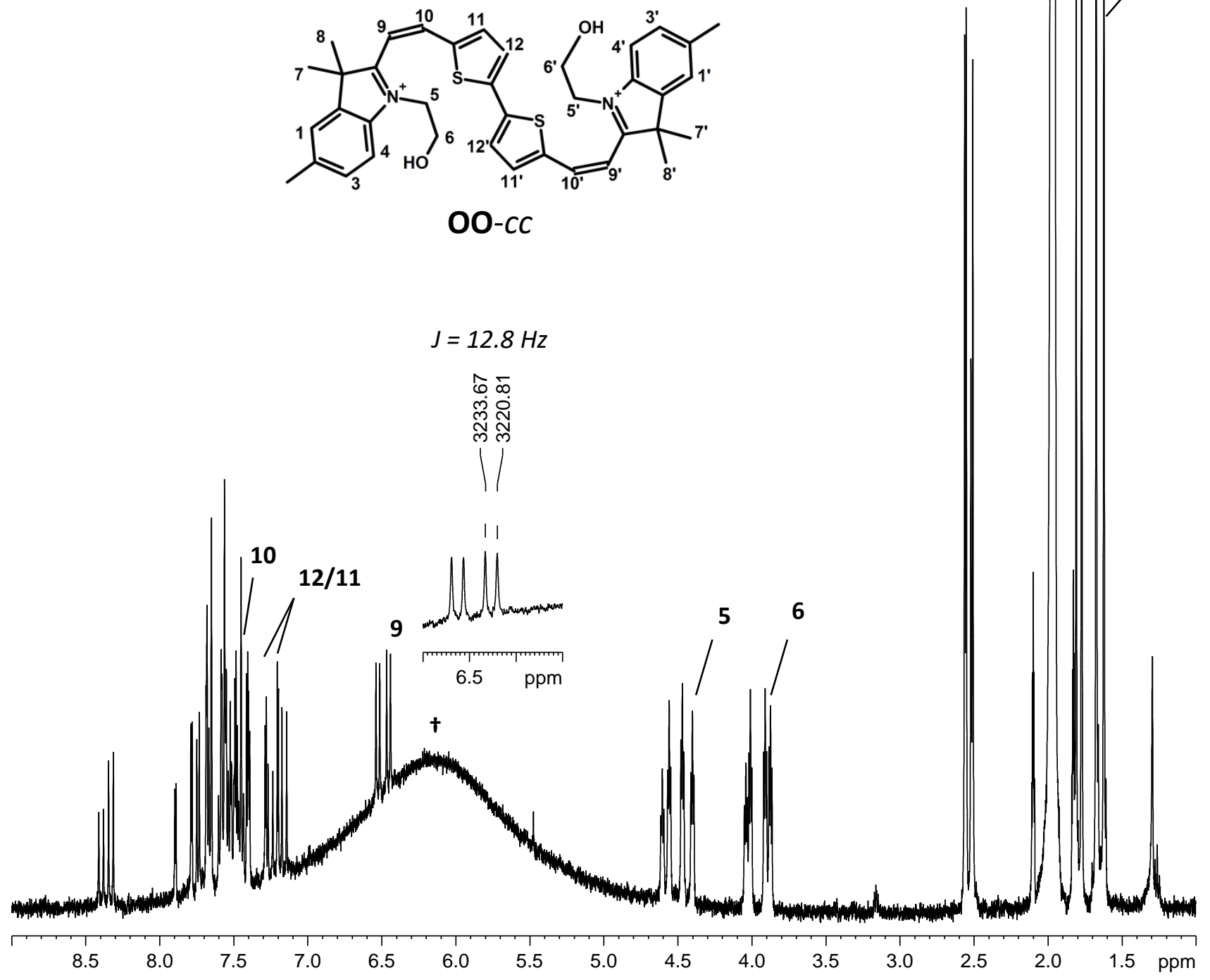

Figure S24 : ${ }^{1} \mathrm{H}$ NMR spectrum of BiBox-Bithio OO-cc in acetonitrile-d $d_{3}$ at room temperature, obtained from 00-tt upon 577 $\mathrm{nm}$ irradiation (7 minutes) - in mixture with 00-tt and 00-tc $-\left({ }^{*}\right)$ solvent $-(t)$ water 


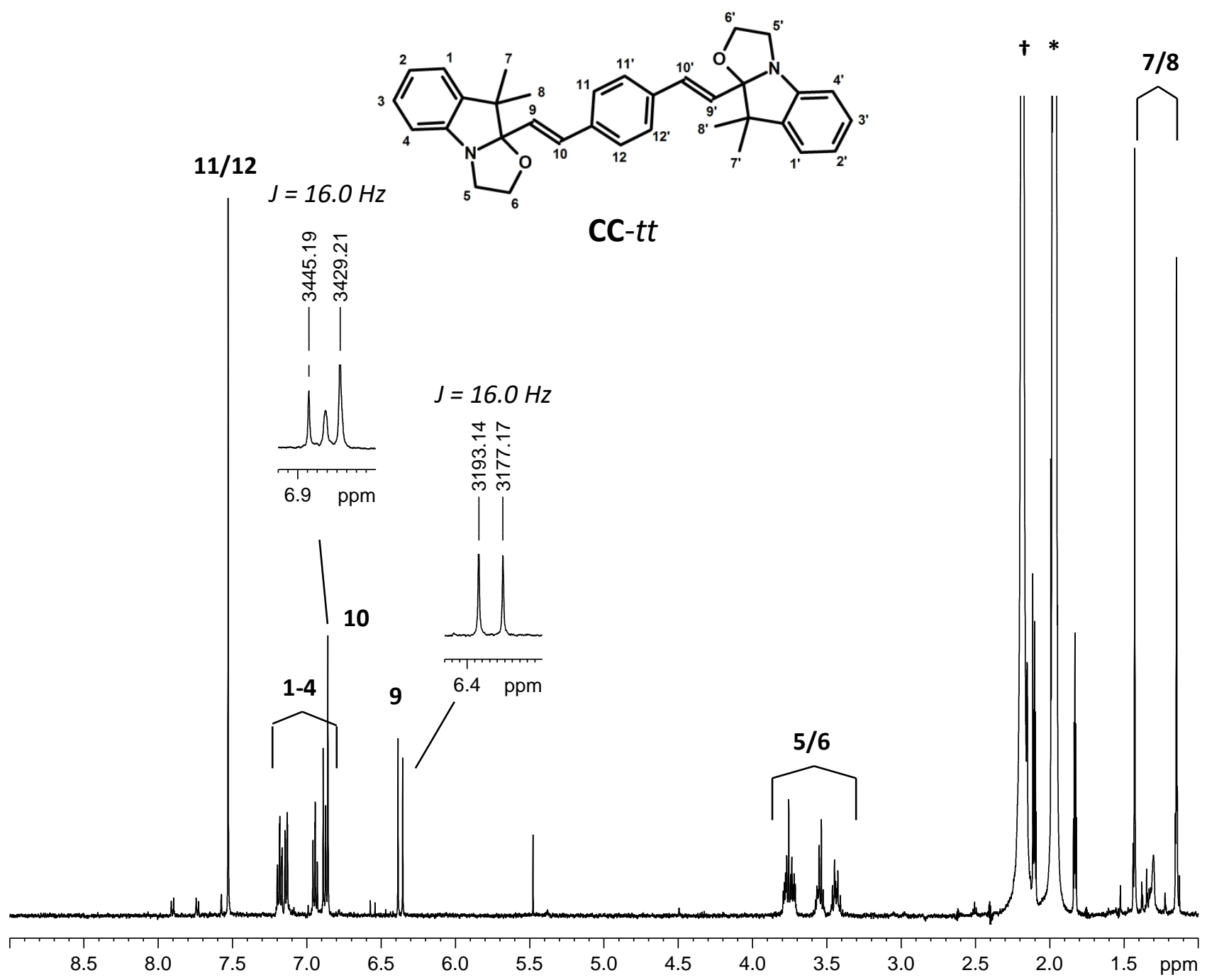

Figure S25: ${ }^{1} \mathrm{H}$ NMR spectrum of BiBox-Phe CC-tt in acetonitrile- $d_{3}$ at room temperature $-\left({ }^{*}\right)$ solvent $-\left({ }^{+}\right)$water 


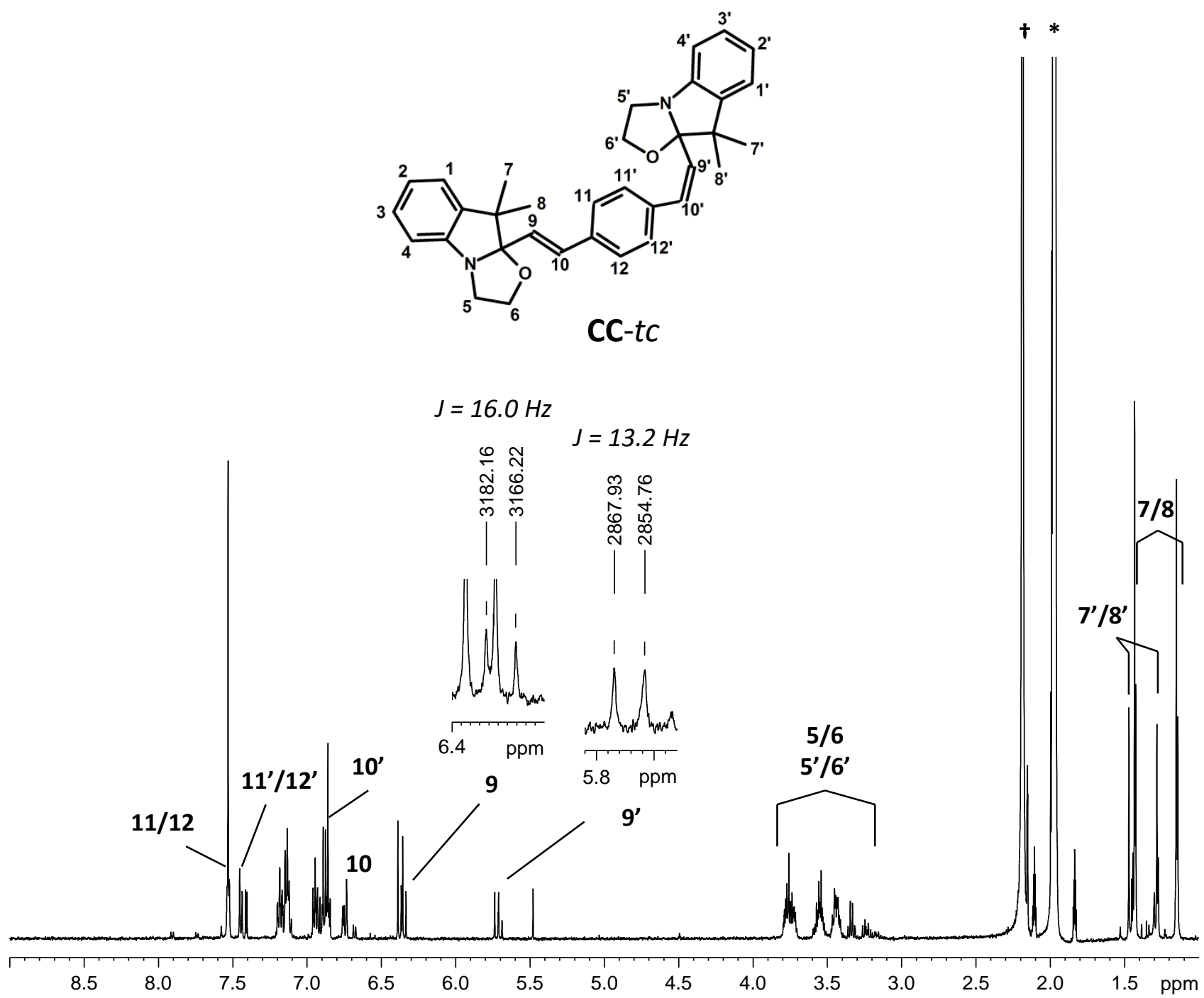

Figure S26: ${ }^{1} \mathrm{H}$ NMR spectrum of BiBox-Phe CC-tc in acetonitrile- $\mathrm{d}_{3}$ at room temperature, obtained from CC-tt upon $436 \mathrm{~nm}$ irradiation (70 minutes) - in mixture with CC-tt $-(*)$ solvent $-(+)$ water 


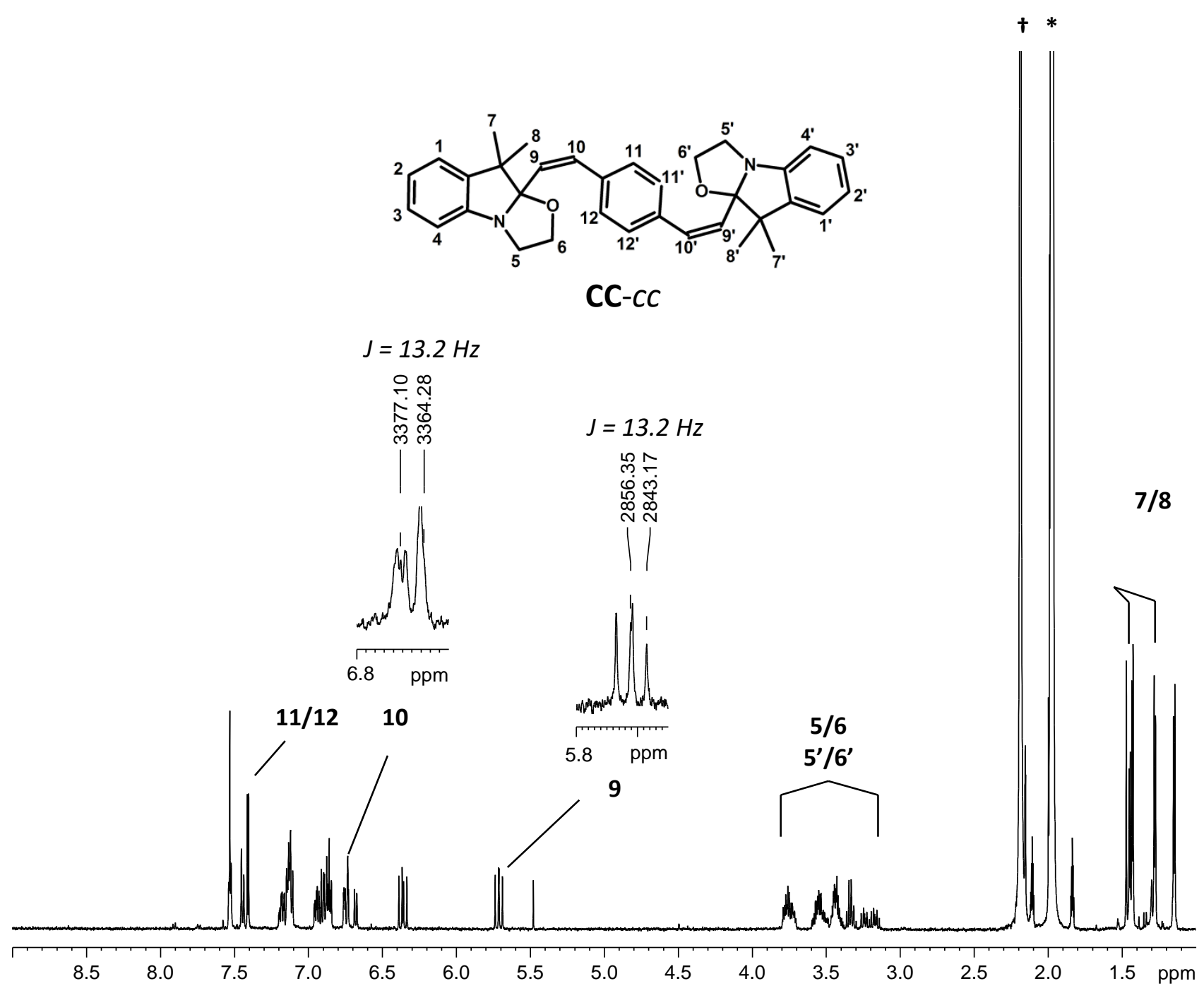

Figure S27 : ${ }^{1} \mathrm{H}$ NMR spectrum of BiBox-Phe CC-cc in acetonitrile- $\mathrm{d}_{3}$ at room temperature, obtained from CC-tt upon $436 \mathrm{~nm}$ irradiation (265 minutes) - in mixture with CC-tt and CC-tc $-(*)$ solvent $-(+)$ water 


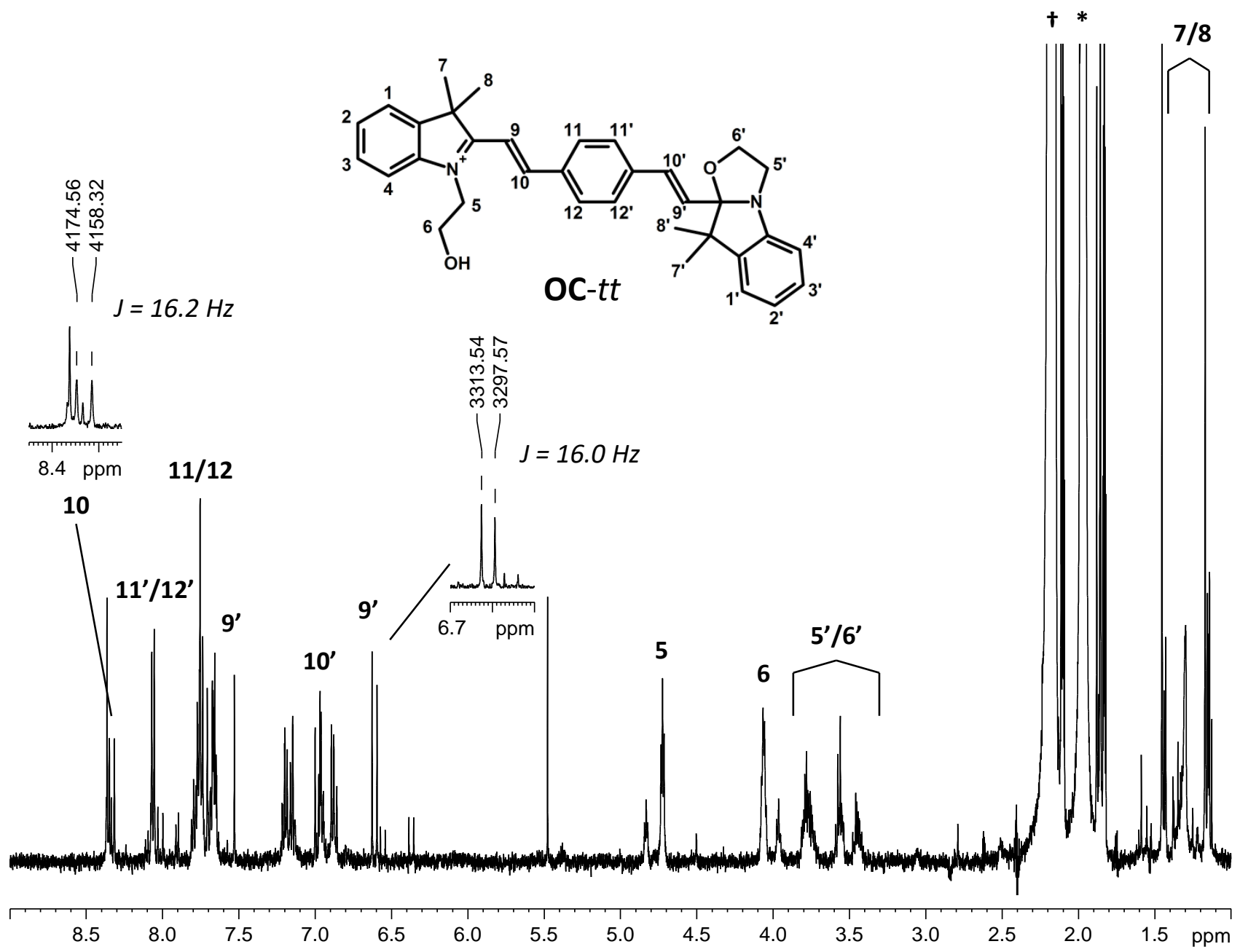

Figure S28 : ${ }^{1} \mathrm{H}$ NMR spectrum of BiBox-Phe OC-tt in acetonitrile- $d_{3}$ at room temperature, obtained from CC-tt upon addition of 1.5 eq. of $\mathrm{DCl}-$ in mixture with CC-tt and OO-tt $-(*)$ solvent $-(t)$ water 


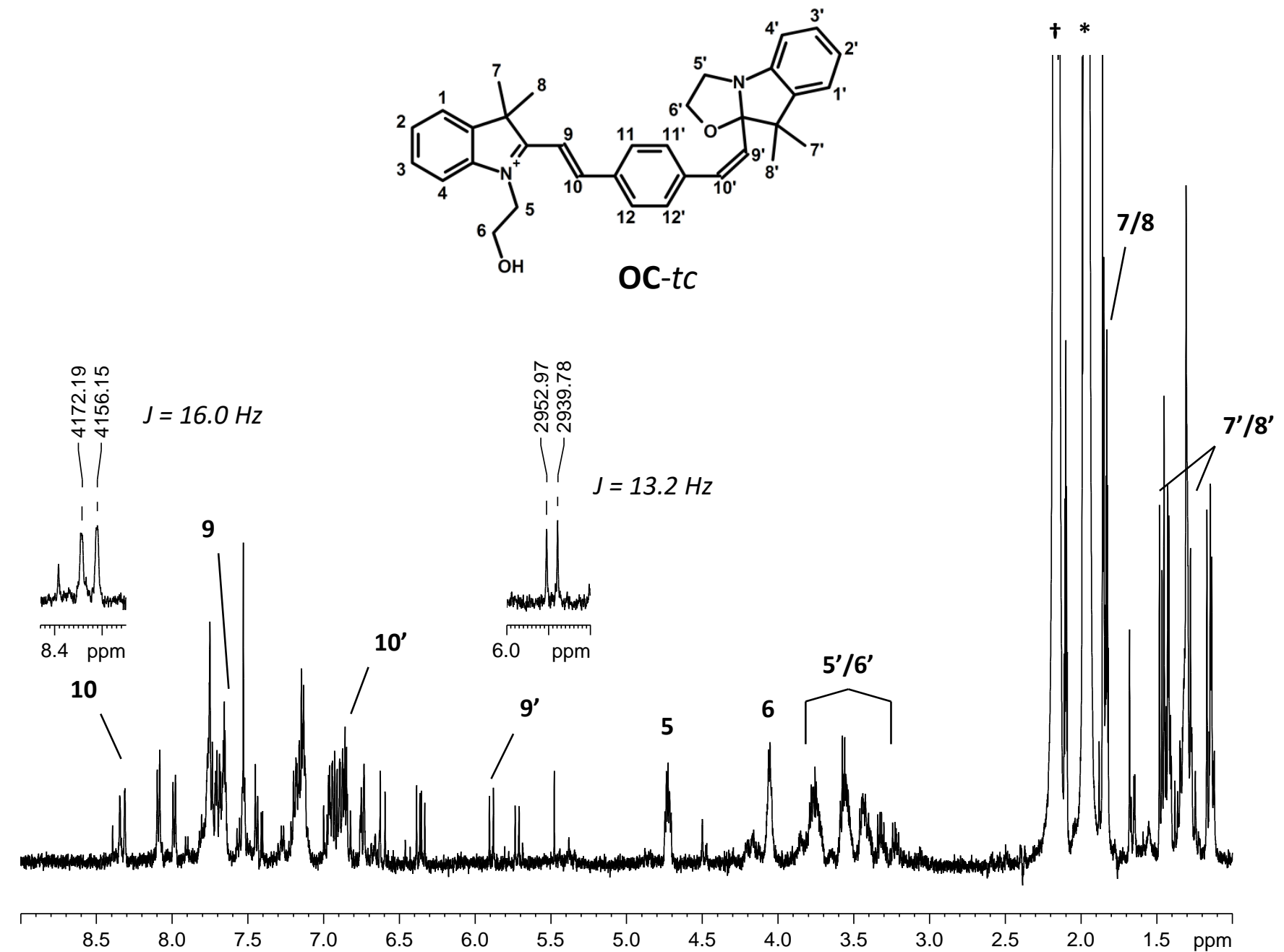

Figure S29: ${ }^{1} \mathrm{H}$ NMR spectrum of BiBox-Phe OC-tc in acetonitrile- $\mathrm{d}_{3}$ at room temperature, obtained from OC-tt upon $436 \mathrm{~nm}$ irradiation (8 minutes) - in mixture with CC-tt, OC-tt and CC-tc $-\left({ }^{*}\right)$ solvent $-(+)$ water 


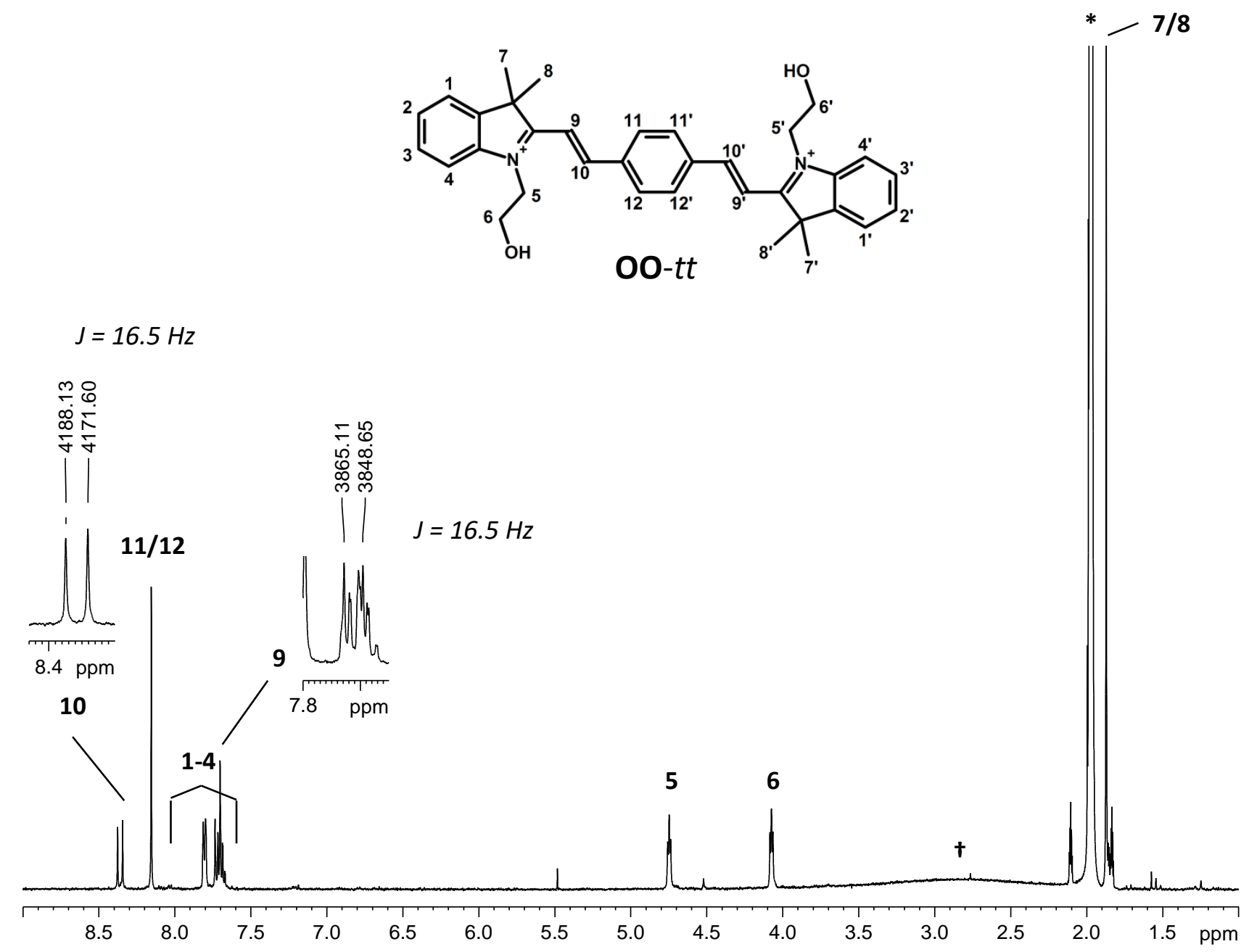

Figure S30 : ${ }^{1} \mathrm{H}$ NMR spectrum of BiBox-Phe OO-tt in acetonitrile-d3 at room temperature, obtained from CC-tt upon addition of TFA vapor in excess $-(*)$ solvent $-(t)$ water 


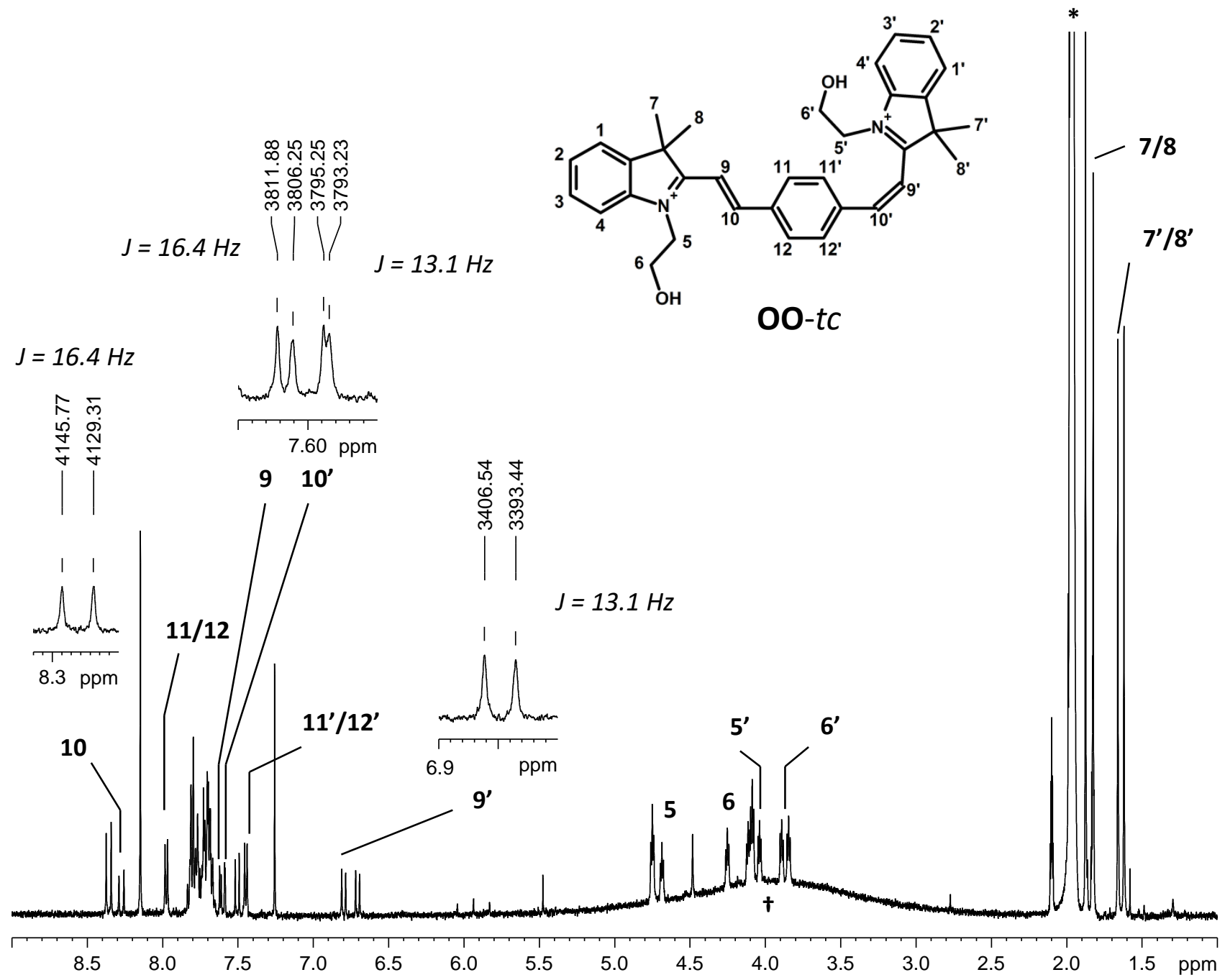

Figure S31 : ${ }^{1} \mathrm{H}$ NMR spectrum of BiBox-Phe OO-tc in acetonitrile- $\mathrm{d}_{3}$ at room temperature, obtained from 00-tt upon $436 \mathrm{~nm}$ irradiation (1.5 minutes) - in mixture with OO-tt and OO-cc $-(*)$ solvent $-(t)$ water 


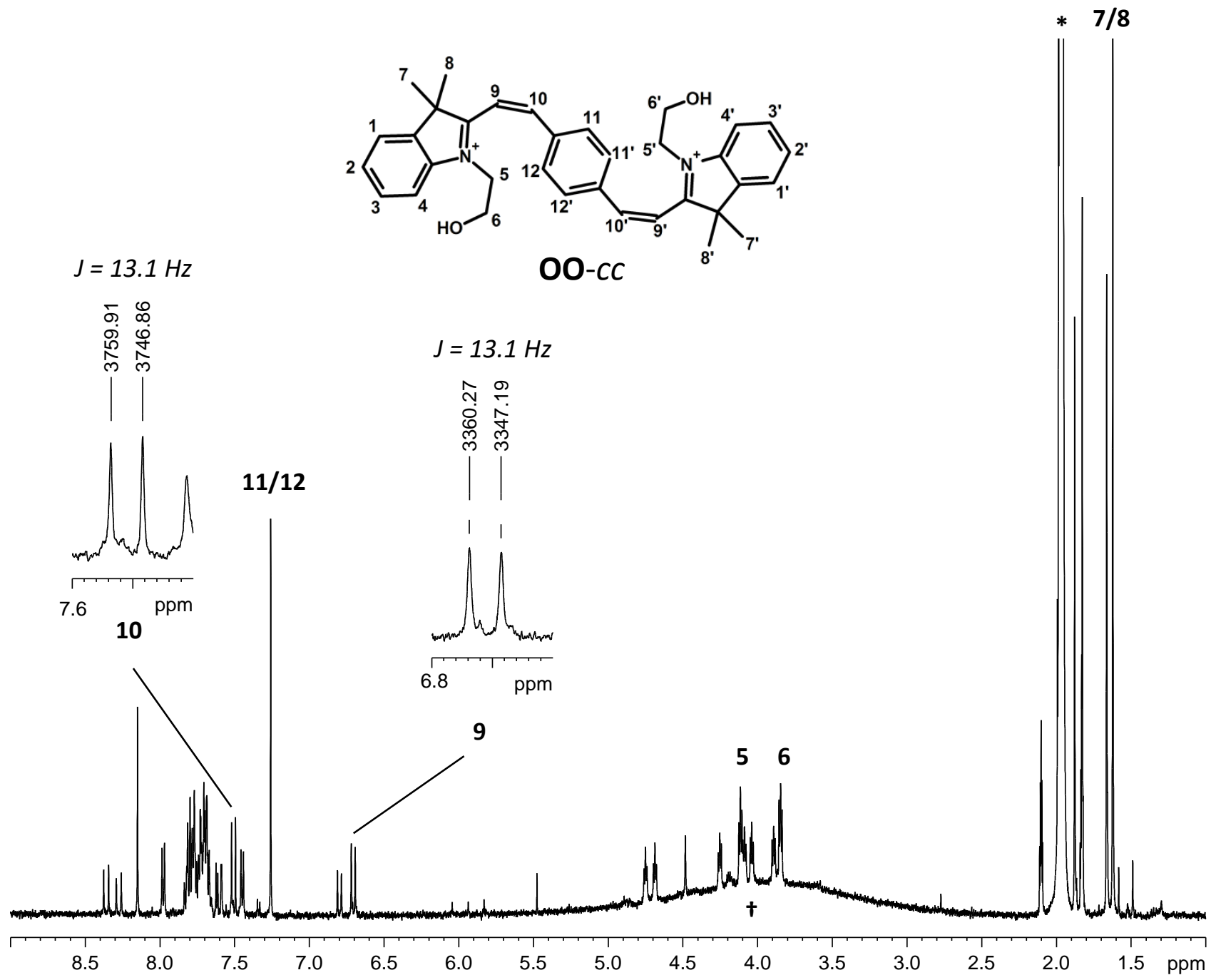

Figure S32 : ${ }^{1} \mathrm{H}$ NMR spectrum of BiBox-Phe 0O-cc in acetonitrile- $\mathrm{d}_{3}$ at room temperature, obtained from 00-tt upon $436 \mathrm{~nm}$ irradiation (6.5 minutes) - in mixture with OO-tt and OO-tc $-\left({ }^{*}\right)$ solvent $-(t)$ water 

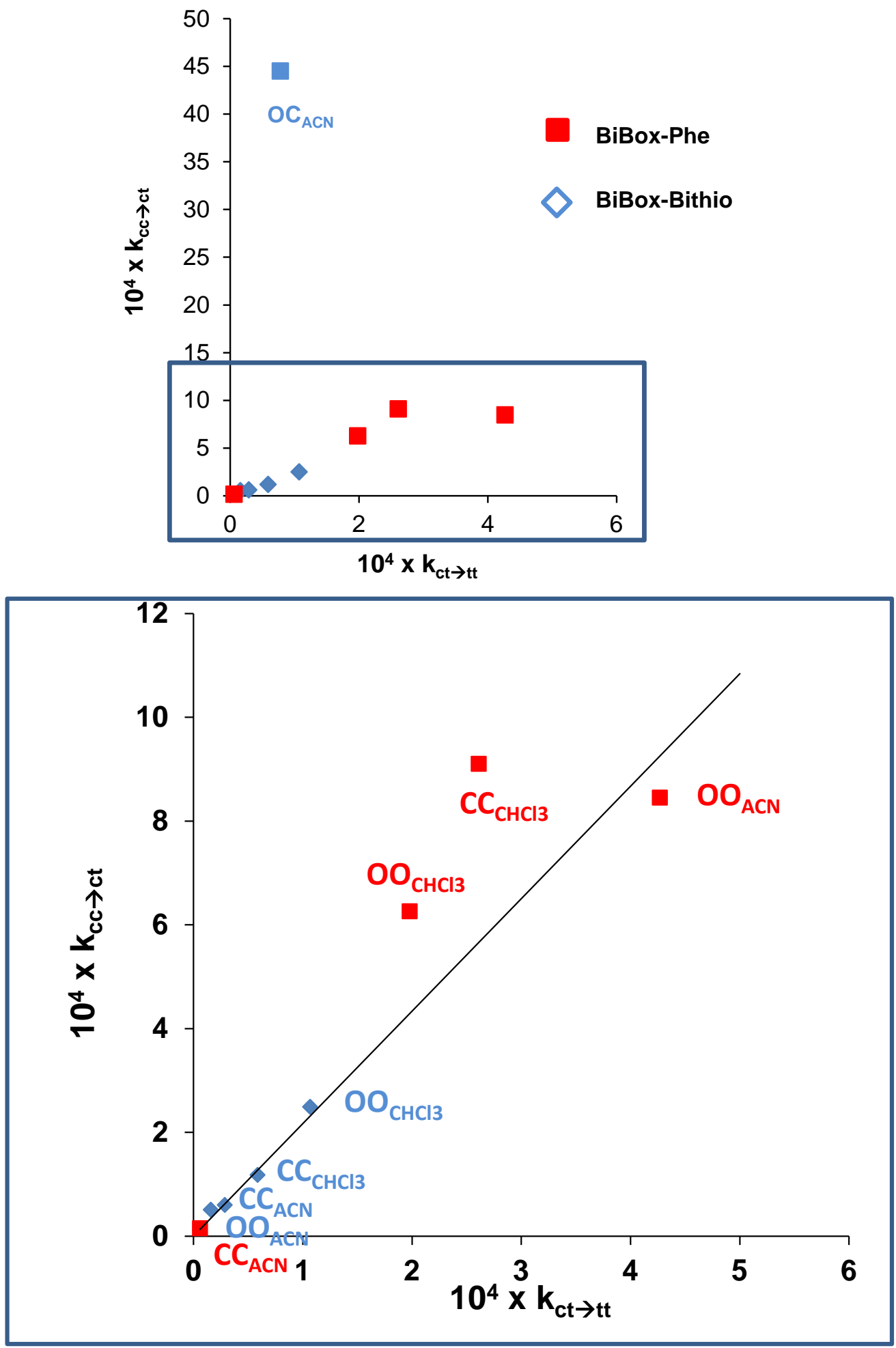

Figure S33 : Variation of the thermal rate constants of relaxation of BiBox : $c c \rightarrow t c=f(t c \rightarrow t t)$ 